\title{
COOPERATIVE MECHANISM OF SELF-REGULATION IN HIERARCHICAL LIVING SYSTEMS*
}

\author{
I. A. LUBASHEVSKY ${ }^{\dagger}$ AND V. V. GAFIYCHUK ${ }^{\ddagger}$
}

\begin{abstract}
.
We study the problem of how a "living" system complex in structure can respond perfectly to local changes in the environment. Such a system is assumed to consist of a distributed "living" medium and a hierarchical "supplying" network that provides this medium with "nutritious" products. Because of the hierarchical organization each element of the supplying network has to behave in a self-consistent way for the system can adapt to changes in the environment.

We propose a cooperative mechanism of self-regulation by which the system as a whole can react perfectly. This mechanism is based on an individual response of each element to the corresponding small piece of the information on the state of the "living" medium. The conservation of flux through the supplying network gives rise to a certain processing of information and the self-consistent behavior of the elements, leading to the perfect self-regulation. The corresponding equations governing the "living" medium state are obtained.
\end{abstract}

Key words. active hierarchical systems, cooperative self-regulation, information self-processing, living media, natural systems

AMS subject classifications. 82C70, 92B05, 92D15, 93A13, 93B52

1. Introduction. "Living" systems and the self-regulation problem. The present paper is devoted to one of the fundamental problems of how complex "living" systems widely met in nature can adapt to changes in the environment. By the term "living" system we mean one that comes into being, provides for itself, and develops pursuing its own goals. This class comprises a great variety of biological and ecological systems. Besides, economic systems can be also placed into such a class because their occurrence, growth, and development is due to self-organization processes. To make the subject of our analysis more clear let us, first, specify the main properties of the systems we deal with.

1.1. Living systems. The elements of such a system, first, should be permanently supplied with external "nutritious" products for their life activities. Second, in order for its elements to subsist special conditions are required. The latter are implemented through these elements making up a certain medium (which will be referred below as to a "living" medium) whose state is controlled by keeping its basic parameters inside certain vital intervals.

As a rule both of these requirements are fulfilled by a supplying network which provides the elements with nutrients as well as controls the living medium state. Since the external products usually penetrate into the system through a common entryway and, then, should be delivered to a great number of elements the flow of these products has to branch many times until it reaches these elements. So the supplying network is to be organized hierarchically and to involve many levels.

Let us, now, present typical examples of such systems. First, this is living tissue, where blood flowing through a vascular network supplies cells with oxygen, nutritious

\footnotetext{
${ }^{*}$ This work was made possible in part by Grants U1I000, U1I200 from the International Science Foundation, Grant 96-02-17576 from the Russion Foundation of Basic Researches and Grant 4.4/103 of Fundation of Fundamental Researches of Ukraine.

${ }^{\dagger}$ Department of Physics, Moscow State University, Vavilova str., 46-92, 117333 Moscow, Russia

${ }^{\ddagger}$ Institute for Applied Problems of Mechanics and Mathematics, National Academy of Sciences of Ukraine, 3b Naukova str. L'viv, 290601, Ukraine
} 
products, etc. At the same time blood withdraws carbon dioxide and other products resulting from the cell life activities, keeping their concentrations inside the vital intervals (see, e.g. [1]). In this way blood flow controls also the tissue temperature [2]. We note that the temperature and the concentration of carbon dioxide (or a similar substance) are major parameters characterizing the living tissue state [1], because their values are directly determined by the life activities of cells. In order to supply with blood each small group of cells the vascular network or, more precisely, its arterial and venous beds are approximately of the tree form and involve many vessels of various lengths and diameters. The regional self-regulation processes keep the carbon dioxide concentration and the temperature inside certain vital intervals only in which living tissue can function normally (see, e.g. [1, 2]).

A similar example is a respiratory system where oxygen going through a hierarchical network of bronchial tubes reaches small capillaries.

Second, large firms are a clear example of economic hierarchical systems. Managers of all functions and of all levels make up a management network [3]. Roughly speaking, the management network controls both the money flow toward the organization low level comprising workers and also the flow of products in the opposite direction. In performing technological processes the wages paid to workers actually transform into the firm products.

The existence of tremendous amount of goods in the market, in contrast to a relatively small number of raw materials shows that there also must be large hierarchical systems supplying the consumers with goods. The goods flow after reaching the consumers transforms into money flow in the opposite direction [ 国 . self-organization phenomena can give rise to trade networks of the tree form [6].

Concerning ecological systems we would like to note that they are also complex in structure and can involve a larger number of "predator-prey" levels [7]. The dynamics of ecosystems is governed by biomass and energy flow on trophic networks and under certain conditions leads to formation of the tree like trophic networks [8].

Keeping in mind the aforementioned examples let us represent a living system as one consisting of a living medium and a supplying network which involves supplying and draining beds of the tree form (Fig. 1.1). (In principle, the two beds can coincide with each other in space.) A transport agent (e.g., blood in living tissue) "flowing" through this network delivers "nutrients" to the living medium and withdraws "life activity" products. The interaction between the transport agent and the living medium (leading to the product exchange) takes place when the transport agent "flows" through the last level branches of the supplying network (in Fig. 1.1 through the branches of level $N$ ).

The concentration of the life activity products will be regarded as the parameter characterizing the living medium state. This is due to the fact that the higher are life activities in intensity, the greater is the concentration of the resulting products. So an increase in this concentration informs the system that the living medium needs a greater amount of "nutrients". In addition, the products resulting from the life activities and remaining in the living medium themselves can depress its functions. Therefore the system has to prevent the concentration of these products exceeding a certain critical value.

The motion of transport agent is accompanied by energy dissipation. So a certain external force should be applied to the system that affects the overall flow of transport agent (for example, in living tissue this is the blood pressure [1, 2]). Further distribution of the transport agent flow over the supplying network is governed by 


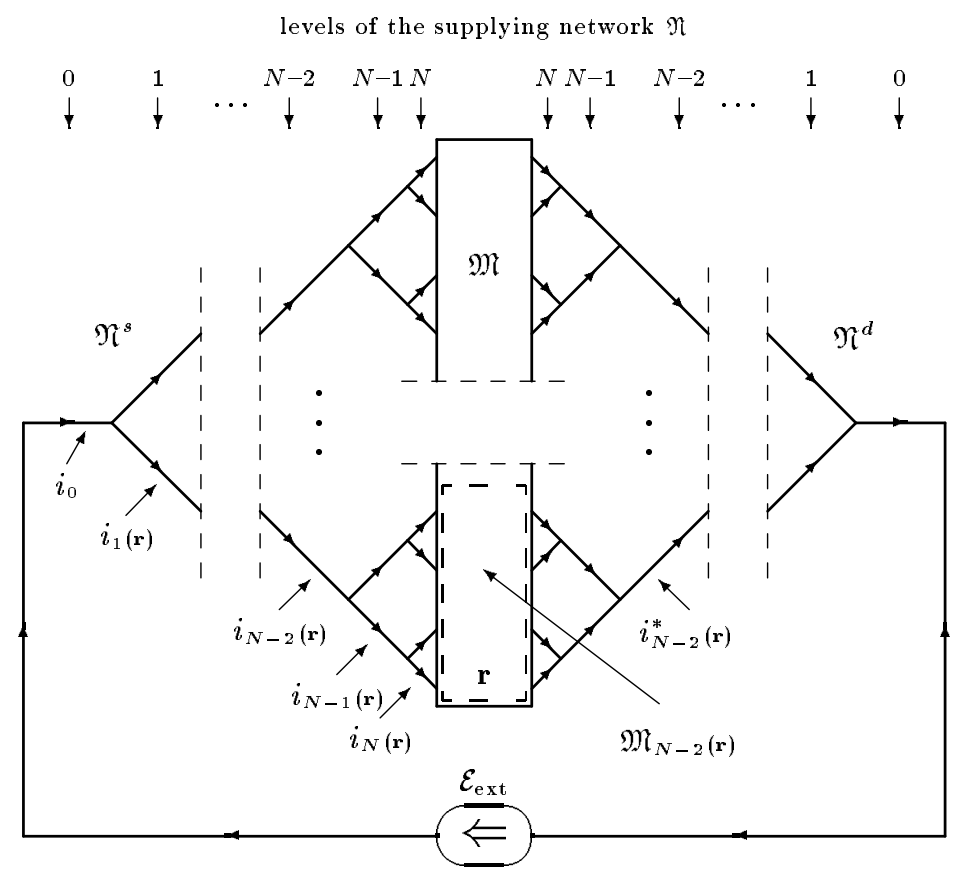

FIG. 1.1. "Living" system structure. (For simplicity is shown the binary network.)

the individual properties of its branches. More precisely, the transport agent flow distribution is controlled by both a certain potential (in living tissue it is the blood pressure) and by the "resistances" of the branches to the transport agent flow. In order to specify the physical regularities of the transport agent flow distribution we may choose various approaches. One of them is Prigogine's principle of minimum entropy production stated in nonequilibrium thermodynamics (see, e.g., 11]). Another is related to Swenson's principle of maximal dissipation. In our analysis the two approaches lead to the same result: the transport agent flows meet an extremum of a certain functional. So, to be defined we will adopt the minimum entropy production principle.

1.2. Self-regulation problem. For different elements of the living medium to fulfill their individual functions independently of one another the supplying network should, at least in the ideal case, have a capacity for controlling the state of the living medium at each its point. This requirement is reduced to the local control of the "perfusion" rate of the transport agent through the living medium. So the independence of the perfusion rate at one point from life activities of elements located at other points is a desirable property which will be referred below as to the perfect self-regulation. In fact, if we ignore diffusion in the living medium solely the perfusion rate of transport agent at a given point will govern functioning of elements at this point: supply them with "nutrients" and withdraw the life activity products. So the perfusion rate should take such a value that provides the optimal conditions for functioning of the elements, depending on their life activities. Therefore if the perfusion rate at one point reacted substantially to the life activities at other points then the living medium elements would interfere with one another and the living 
system could lose its capacity for adapting. The existence of a great variety of living systems in nature enables us to think that such a nonlocal interaction is suppressed or, at least, depressed to a certain degree. However, in trying to describe how this selfregulation can be implemented, i.e. its particular mechanism we meet the following fundamental problem.

The perfusion rate of the transport agent at a given point $\mathbf{r}$ is certain to be determined by its flow through the corresponding last level branch $i_{N}(\mathbf{r})$ of the supplying network (Fig. 1.1). However, this flow in turn depends on the transport agent flow through the branch $i_{N-1}(\mathbf{r})$ of the previous level connected with the branch $i_{N}(\mathbf{r})$ and so on up to the stem $i_{0}$ (the branch of zeroth level). In this way we find a path $\mathbb{P}(\mathbf{r})=\left\{i_{0}, i_{1}(\mathbf{r}), \ldots, i_{N-1}(\mathbf{r}), i_{N}(\mathbf{r})\right\}$ on the supplying network through which the transport agent flow reaches a small neighborhood of the point $\mathbf{r}$. Let us consider the transport agent flow through one of these branches, for example, a branch $i_{n}(\mathbf{r})$ of level $n<N$ (in Fig. 1.1 it is the branch $i_{N-2}(\mathbf{r})$ ). This branch supplies with "nutrients" not only elements in the vicinity of the point $\mathbf{r}$ but also all of the elements located inside a certain domain $\mathfrak{M}_{n}(\mathbf{r})$ (inside the domain $\mathfrak{M}_{N-1}(\mathbf{r})$ in Fig. 1.1) and controls the living medium state in this domain as a whole. So the required flow of the transport agent through the branch $i_{n}(\mathbf{r})$ is specified by the life activities of all the elements belonging to the domain $\mathfrak{M}_{n}(\mathbf{r})$ and a change in the life activities of one of them inevitably will cause this flow to vary.

Since the external force applied to the system controls only the overall flow the specific distribution of the transport agent flow over the supplying network is governed by the "resistances" of all the branches. Under such conditions, in general, a change in the transport agent flow through the branch $i_{n}(\mathbf{r})$ (for example, because of variations in its "resistance") causes the transport agent flow to alter in all the branches of higher levels that are connected with $i_{n}(\mathbf{r})$, in particular, in a last level branch $i_{N}\left(\mathbf{r}^{\prime}\right)$ leading to a point $\mathbf{r}^{\prime} \in \mathfrak{M}_{n}(\mathbf{r})$ and $\mathbf{r}^{\prime} \neq \mathbf{r}$. So, in principle, a change in the life activities of elements near the point $\mathbf{r}$ will lead via the branch $i_{n}(\mathbf{r})$ to variations in the perfusion rate of transport agent at all the points belonging to the domain $\mathfrak{M}_{n}(\mathbf{r})$. Since the domains $\left\{\mathfrak{M}_{n}(\mathbf{r})\right\}$ increase in size and tend to the total space of the living medium as we pass on the supplying bed from the last level to its stem this effect is nonlocal substantially.

Therefore special conditions are required for the self-regulation to be perfect because in the general case the perfusion rate of the transport agent is determined by the life activities of all the elements.

In other words for the perfect self-regulation to occur the "resistances" of all the branches should vary in a self-consistent way. In this place we actually meet the fundament problem mentioned above: what governs such variations of the branch "resistances"? For a large living system it is unlikely that any one of its elements can possess the whole information required of governing the perfect response to changes in the environment and, so, in the life activities of living medium. This is due to the fact that such a control requires processing of a great amount of information characterizing the living medium state on all spatial scales (i.e. at all the levels of supplying network). In particular, in living organisms local alterations in homeostasis seem to be controlled by regional mechanisms of self-regulation rather than the central nervous system [1]. Besides, none of these elements can individually control transport agent flow through the supplying network because of the mass conservation at its branching points.

The aforementioned allows us to assume that the perfect self-regulation can be 
implemented through cooperative mechanisms. By this scenario each branch of the supplying network receives the corresponding small piece of the information on the living medium state and their reaction to this information gives rise to the desired redistribution of the transport agent flow over the supplying network. In other words, the branch "resistances" vary in such a self-consistent way that enables the supplying network to provide, for example, an additional amount of "nutrients" for the living medium elements which have a need for this and not to disturb transport agent flow at other points of the living medium.

However, for the cooperative mechanism of perfect self-regulation to come into being, first, a certain self-processing of the information is required which enables the branches to react adequately, depending on their place in the supplying network. Second, the physical properties of the supplying network should give rise to the cooperative effect of variations in the branch "resistances" on the transport agent flow redistribution leading to the desired results.

The purpose of the present paper is to show that these requirements can be fulfilled and so a cooperative mechanism of perfect self-regulation in living systems can exist. We will demonstrate that there is a required self-processing of information and the physics of transport agent motion ensures its proper redistribution over the supplying network. Beforehand we can say that it is the mass conservation at the nodes of supplying network and its hierarchical organization those bring into being the information self-processing. This information self-processing is implemented through measuring the concentration of the life activity products inside each branch of the draining bed. Due to the interaction with the living medium the transport agent saturates with the life activity products when it flows through the last level branches. Then the transport agent moves through the draining bed from higher hierarchy levels to lower ones and, so, at the nodes its smaller streams unite into larger streams. Therefore, due to the mass conservation the concentration $\theta_{i}$ of life activity products inside, for example, a branch $i$ is actually equal to the concentration of life activity products inside the living medium averaged over the domain $\mathfrak{M}_{i}$ which is drained as a whole by this branch. So the value of $\theta_{i}$ aggregates the information on the living medium state in the domain $\mathfrak{M}_{i}$ as a whole and, thus, can play the role of the information piece needed for this branch and the corresponding branch of the supplying bed to react properly to variations in the life activities of the living medium. The correspondence between the branches of the supplying and draining beds is illustratied in Fig. 1.1 by the pair $i_{N-2}(\mathbf{r}), i_{N-2}^{*}(\mathbf{r})$.

It should be noted that developing this model we actually have kept in mind one of the possible mechanisms of self-regulation in living tissues. Life activities of cellular tissue gives rise to variations in the carbon dioxide concentration in it and, so, in venous blood. Receptors embedded into the vein walls through a regional nervous system govern the expansion or contraction of the corresponding arteries (see, e.g., [1, 21).

Below, at first, we will analyze in detail the model for a living system with a regular supplying network. This network is organized in such a manner that the perfusion rate of transport agent be the same at every point of the living medium, all other factors being equal. In particular, we will develop a technique that not only enables us to study the perfect response of living system to changes in the living medium state but also can be used in investigations of its more complex behavior. Then we will show that the perfect self-regulation can also occur in living systems with nonregular supplying networks of the general form. 
2. Mathematical model for living system with regular supplying network. Let us consider a system consisting of a living medium $\mathfrak{M}$ and a hierarchical supplying network $\mathfrak{N}$ similar to that shown in Fig. 1.1. The network $\mathfrak{N}$ involves supplying $\left(\mathfrak{N}^{s}\right)$ and draining $\left(\mathfrak{N}^{d}\right)$ beds of the tree form (illustrated in Fig. 1.1 by the left and right-hand side parts) and an external element $\mathcal{E}_{\text {ext }}$ joining the tree stems. Transport agent flowing through the former bed supplies the living medium $\mathfrak{M}$ with "nutrients". At the same time transport agent withdraws life activity products from the living medium through the draining bed. For simplicity we assume that the living medium domain $\mathfrak{M}$ is a $d$-dimensional cube of edge $l_{0}$ and the geometry of both the beds is the same.

The supplying and draining beds can be represented as the collections of groups of branches $\{i\}$ belonging to one level: $\mathfrak{N}^{s}=\left\{\mathbb{L}_{n}^{s}\right\}_{n=0}^{N}, \mathfrak{N}^{d}=\left\{\mathbb{L}_{n}^{d}\right\}_{n=0}^{N}$, where $\mathbb{L}_{n}^{s(d)}=\left\{i: i \in \mathfrak{N}^{s(d)}\right.$ and $i \in$ level $\left.n\right\}$. Below we will use the symbol $\mathbb{L}_{n}$ instead of $\mathbb{L}_{n}^{s}$ or $\mathbb{L}_{n}^{d}$ where it does not lead to misunderstanding or gives the same results due to the mirror symmetry of the supplying and draining beds. In these terms the embedding of the supplying network $\mathfrak{N}$ (as the collection of branches $\{i\}$ ) into the living medium matches the partition of the domain $\mathfrak{M}$ into the following collection of cubes $\left\{\left\{\mathfrak{M}^{i}\right\}_{i \in \mathbb{L}_{n}}\right\}_{n=0}^{N}$.

For each level $n$ the group $\mathbb{M}_{n}=\left\{\mathfrak{M}^{i}\right\}_{i \in \mathbb{L}_{n}}$ involves $2^{\text {nd }}$ equal disjoint cubes of edge $l_{n}=l_{0} 2^{-n}$ that together compose the whole living medium domain:

$$
\mathfrak{M}=\bigcup_{i \in \mathbb{L}_{n}} \mathfrak{M}^{i} \quad \text { and } \quad \mathfrak{M}^{i} \bigcap \mathfrak{M}^{j}=\emptyset \quad \text { if } \quad i \neq j, \quad i, j \in \mathbb{L}_{n}
$$

For any two levels $n$ and $m<n$ each domain $\mathfrak{M}^{i} \in \mathbb{M}_{n}$ belongs to one of the domains of the group $\mathbb{M}_{m}$ and, thus, each domain $\mathfrak{M}^{j} \in \mathbb{M}_{m}$ can be represented as a certain union of the domains of group $\mathbb{M}_{n}$

$$
\mathfrak{M}^{j}=\bigcup_{i \in \mathbb{L}_{n}^{j}} \mathfrak{M}^{i}
$$

For zeroth level $\mathfrak{M}^{i_{0}}=\mathfrak{M}$. Below the cubes of the group $\mathbb{M}_{n}=\left\{\mathfrak{M}^{i}\right\}_{i \in \mathbb{L}_{n}}$ will be also called fundamental domains of level $n$ and those of the last level $N$ will be also referred to as elementary domains.

The supplying and draining beds are embedded into the living medium in such a way that every branch $i$ of a given level $n$ supplies (or drains) a certain domain $\mathfrak{M}^{i} \in \mathbb{M}_{n}$ as a whole. Each elementary domain is bound up with one of the last level branches. The last level number $N$ is assumed to be much larger than unity: $N \gg 1$, and the length $l_{N}$ may be regarded as an infinitely small spatial scale. Transport agent flow is directed from lower to higher levels on the supplying bed and in the opposite direction on the draining bed.

Since the geometry of both the beds is assumed to be the same, we specify it for the supplying bed only. The stem of this bed (Fig. 2.1) goes into the cube $\mathfrak{M}$ through one of its corners and reaches the cube center $O_{0}$, where it splits into $g=2^{d}$ branches of the first level. Each branch of the first level reaches a center $O_{1}$ of one of the $g$ fundamental domains of the first level. At the centers $\left\{O_{1}\right\}$ each of the first level branches in turn splits into $g$ second level branches. Then this process is continued in a similar way up to level $N$. The branches of the last level $N$ are directly connected 

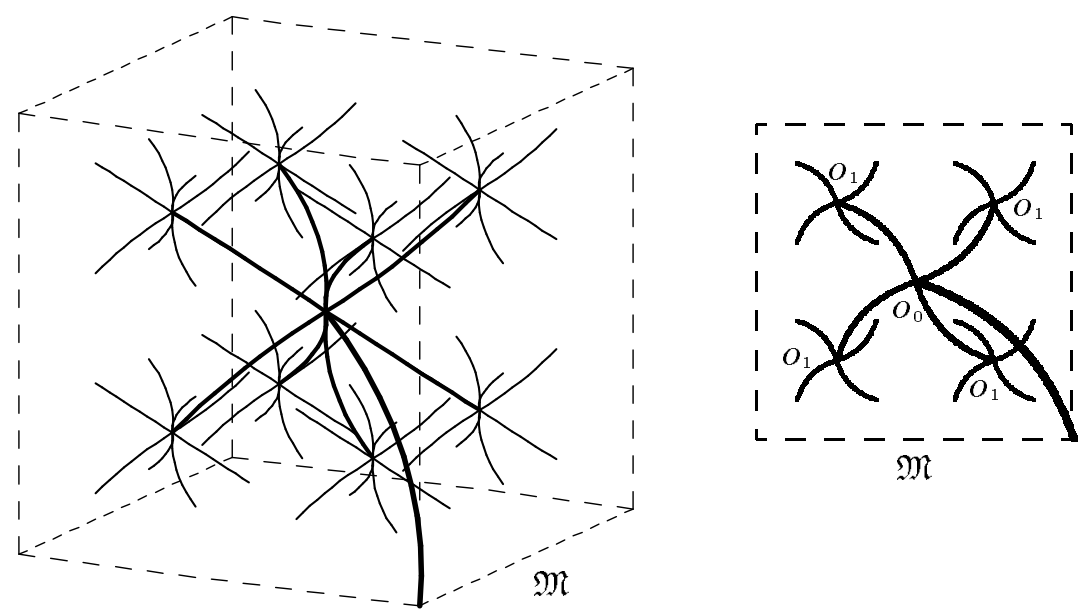

FIG. 2.1. Fragments of the supplying network architectonics for the two- and three-dimensional living medium

with living medium $\mathfrak{M}$. It should be noted that in this model the length $\ell_{n}$ of branches belonging to level $n$ is estimated by the expression $\ell_{n} \sim \sqrt{d} l_{n}=\sqrt{d} 2^{-n}$ and smaller is a branch, to a higher level belongs it.

Transport agent does not interact with the living medium $\mathfrak{M}$ during its motion through the branches except for the last level ones. When the transport agent reaches one of the last level branches of the supplying bed, for example, branch $i_{N}$ the "nutritious" products carried with it uniformly spread over the elementary domain $\mathfrak{M}_{N}^{i}$ containing the branch $i_{N}$. At the same time transport agent is saturated with the life activity products, withdrawing them from the domain $\mathfrak{M}_{N}^{i}$. In this way the life activity products going with the transport agent through the draining bed leave the system.

The state of the medium $\mathfrak{M}$ is described by a certain field $\theta(\mathbf{r}, t)$ being the dimensionless concentration of life activity products. Dynamics of the field $\theta(\mathbf{r}, t)$ is governed by the volumetric generation $q(\mathbf{r}, t)$ resulting from the life activities, the dissipation because of draining the living medium, and the diffusion. In other words the field $\theta(\mathbf{r}, t)$ is considered to evolve according to the equation

$$
\frac{\partial \theta}{\partial t}=D \nabla^{2} \theta+q-\theta \eta
$$

where $D$ is the diffusivity and $\eta(\mathbf{r}, t)$ is the perfusion rate of transport agent. Since a branch $i_{N}$ of the last level supplies the elementary domain $\mathfrak{M}_{N}^{i}$ of the living medium as a whole the transport agent flow $J_{i_{N}}$ through the branch $i_{N}$ and the perfusion rate $\eta(\mathbf{r}, t)$ are related by the expression

$$
J_{i_{N}}=\int_{\mathfrak{M}_{N}^{i}} \eta(\mathbf{r}) d \mathbf{r} .
$$

The distribution of the transport agent flow $\left\{J_{i}\right\}$ over the network $\mathfrak{N}$ obeys the mass conservation at its nodes $\{\mathfrak{b}\}$ 


$$
\left(J_{i}\right)_{\text {in }}=\sum_{i \in \mathfrak{b}}^{\text {out }} J_{i} \quad \text { or } \quad \sum_{i \in \mathfrak{b}}^{\text {in }} J_{i}=\left(J_{i}\right)_{\text {out }},
$$

where $J_{i}$ is the transport agent flow through a branch $i$ going in or out of the node $\mathfrak{b}$ and the sums run over all the branches $i \in \mathfrak{b}$ leading from or to this node for the supplying and draining beds, respectively. Provided the field $\eta(\mathbf{r}, t)$ is given expressions (2.4) and (2.5) completely specify the quantities $\left\{J_{i}\right\}$.

The last term in equation (2.3) implies that the transport agent when going through the branch $i_{N}$ is saturated with the life activity products up to their concentration in the elementary domain $\mathfrak{M}_{N}^{i}$. Then these products moves with the transport agent through the draining bed without exchange with the living medium. Therefore, we also ascribe to the transport agent going through the draining bed the collection of variables $\left\{\theta_{i}\right\}$ measuring the concentration of the life activity products inside the branches $\left\{i \in \mathfrak{N}^{d}\right\}$ and regard the value $J_{i} \theta_{i}$ as the flow of these products in the branch $i$. For a branch $i_{N}$ of the last level we write

$$
J_{i_{N}} \theta_{i_{N}}=\int_{\mathfrak{M}_{N}^{i}} \theta(\mathbf{r}) \eta(\mathbf{r}) d \mathbf{r} .
$$

and assuming the conservation of the life activity products at the nodes of the draining bed we get

$$
\sum_{i \in \mathfrak{b}}^{\text {in }} J_{i} \theta_{i}=\left(J_{i} \theta_{i}\right)_{\text {out }} .
$$

Equation (2.3) among with expressions (2.4) and (2.6) describe the dynamics of the state of the the living medium and its interaction with transport agent. Equations (2.5) and (2.7) reflect the general laws of transport phenomena in the supplying and draining beds.

Let us, now, specify the regularities governing the transport agent flow through the network $\mathfrak{N}$. First, provided the characteristic parameters of branches are fixed the transport agent flow patter $\left\{J_{i}\right\}$ is assumed to meet the minimum condition of the total energy dissipation due to transport agent motion:

$$
\mathbb{D}\left\{J_{i}\right\} \Rightarrow \min _{\left\{J_{i}\right\}}
$$

subject to relationships (2.5), where the total rate of energy dissipation is given by the expression

$$
\mathbb{D}\left\{J_{i}\right\}=\frac{1}{2} \sum_{i} R_{i} J_{i}^{2}-J_{0} \mathcal{E}_{\text {ext }} .
$$

Here $R_{i}$ is the kinetic coefficient characterizing the energy dissipation during the motion of transport agent through the branch $i, \mathcal{E}_{\text {ext }}$ is the external force causing the 
transport agent motion through the network $\mathfrak{N}$ as a whole, $J_{0}$ is the transport agent flow through the tree stems, and the sum runs over all the branches of the network $\mathfrak{N}$. It should be noted that previously by the term "branch resistances" we have exactly meant the given kinetic coefficients because they do play the role of the true branch resistances to transport agent flow as it will be shown below in the next section.

Second, in this model it is the coefficients $\left\{R_{i}\right\}$ those characterize the individual effect of the branches on the transport agent flow. So the system response to changes in the living medium state, namely, to variations in the field $\theta(\mathbf{r}, t)$ (see Introduction) is represented as time variations of the coefficients $\left\{R_{i}\right\}$. Since the supplying and draining beds have been assumed to be equivalent in architectonics the flow pattern on these beds $\left\{J_{i}\right\}$ as well as the coefficient collection $\left\{R_{i}\right\}$ are set to be the mirror images of each other within reversing the flow direction. The latter allows us to confine our description of the system response to the draining bed.

As mentioned in Introduction the pattern $\left\{\theta_{i}\right\}$ can be treated as the aggregated information on the living medium state on all the spatial scales. In particular, the variable $\theta_{i}$ corresponding to the branch $i$ characterizes the state of the living medium in the fundamental domain $\mathfrak{M}^{i}$ as a whole. Therefore we assume that for each branch, for example, a branch $i$ of level $n$ time variations in the coefficient $R_{i}$ are directly controlled by the variable $\theta_{i}$ assigned to this branch. Under the steady-state conditions this means that the coefficient $R_{i}$ is an explicit function of the variable $\theta_{i}$ identical for all the branches of one level $n$, i.e. $R_{i}=R_{n}\left(\theta_{i}\right)$. The general properties of the $R_{n}(\theta)$ dependence are actually determined by the fact that the transport agent flow through the network $\mathfrak{N}$ should grow as the variables $\left\{\theta_{i}\right\}$ increase. Indeed, let changes in the environment cause the life activities of the living medium to grow in intensity. The latter leads immediately to an increase in the concentration $\theta(\mathbf{r}, t)$ of life activity products inside the living medium, causing an increase in the variables $\left\{\theta_{i}\right\}$. The higher is the life activity intensity, the greater is the amount of "nutrients" needed for the living medium. Therefore, under such conditions the transport agent flow through the network $\mathfrak{N}$ must increase too. In other words, an increase in the variables $\left\{\theta_{i}\right\}$ should give rise to an increase of transport agent flow. The less is the coefficient $R_{i}$, the greater is the transport agent flow that can go through the branch $i$, all other factors being equal. Thus, the function $R_{n}(\theta)$ must be decreasing with respect to the variable $\theta$. In addition, there should be a certain critical concentration $\theta_{c}$ of the life activity products showing the upper boundary of the vital interval for the field $\theta(\mathbf{r}, t)$, i.e. the maximum of the allowable concentration of the life activity products in the living medium. The system has to prevent the field $\theta(\mathbf{r}, t)$ exceeding the value $\theta_{c}$ as much as it can. Otherwise, the system can lose the capability for adapting. In particular, if the field $\theta(\mathbf{r}, t)$ reaches the boundary of the vital interval at all the points of the living medium and, consequently, all the variables $\left\{\theta_{i}\right\}$ come close to the critical value $\theta_{c}$ (as it follows from (2.5)-(2.7)) all the branches should exhaust their capability for decreasing the coefficients $\left\{R_{i}\right\}$.

Taking the aforesaid into account we represent the $R_{n}(\theta)$ dependence in terms of

$$
R_{n}(\theta)=R_{n}^{0} \phi\left(\frac{\theta}{\theta_{c}}\right)
$$

where $R_{n}^{0}$ is a constant equal to $R_{n}(\theta)$ at $\theta=0$ and $\phi(x)$ is a certain universal function of $x=\theta / \theta_{c}$. The characteristic form of the $\phi(x)$ dependence is shown in Fig. 2.2a.

For the transport network $\mathfrak{N}$ to be able to react properly to local variations in the life activities the transport agent flow should be governed by branches of all the 

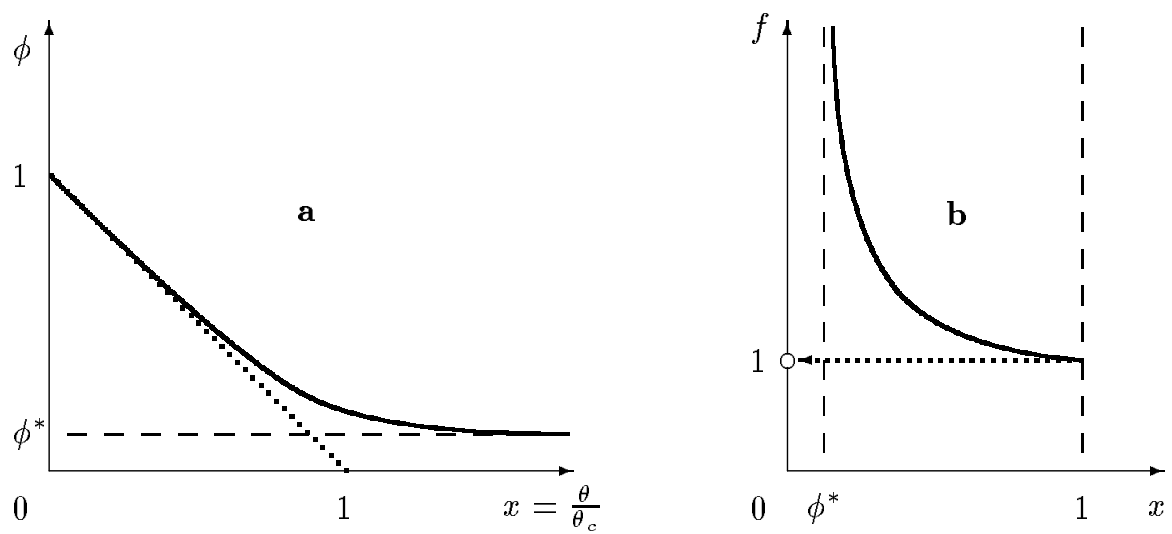

FIG. 2.2. Functions describing the branch response. (The dotted lines correspond to the ideal case.)

levels. As will be seen below this condition allows us to represent the dependence of the value $R_{n}^{0}$ on the level number $n$ in the form

$$
R_{n}^{0}=R_{0} g^{n} \rho(n)
$$

where $R_{0}$ is a certain constant and $\rho(n)$ is a smooth function of $n$ such that $\rho(0)=1$ and formally $\rho(n) \rightarrow 0$ as $n \rightarrow \infty$.

Concluding the description of the system response we also take into account a possible time delay of the branch response to variations in the variables $\left\{\theta_{i}\right\}$ and represent the evolution equation for the kinetic coefficient $R_{i}$ of branch $i$ belonging to level $n$ as

$$
\tau_{n} \frac{d R_{i}}{d t}+\left(R_{i}-R_{n}^{0}\right) f\left(\frac{R_{i}}{R_{n}^{0}}\right)=-\frac{\theta_{i}}{\theta_{c}} R_{n}^{0},
$$

where $\tau_{n}$ is the time delay depending solely on the level number $n$. This form of equation (2.12) enables us to regard the term $\theta_{i} / \theta_{c}$ as a dimensionless signal generated by receptors imbedded into the branch $i$. As follows from (2.10) and (2.12) the functions $f(x)$ and $\phi(x)$ are related by the expression

$$
[1-\phi(x)] f[\phi(x)]=x .
$$

The behavior of the function $f(x)$ is displayed in Fig. 2.2 b.

In the context of the stated model the problem of self-regulation is reduced to finding an expression relating the perfusion rate $\eta(\mathbf{r}, t)$ of the transport agent to the field $\theta(\mathbf{r}, t)$ :

$$
\eta=\hat{\Phi}\{\theta\} \quad \text { or } \quad \eta=\hat{\Phi}\{\theta, \eta\} .
$$

In general, this expression is of the functional form, i.e. is nonlocal in space and only under specific conditions can relate the fields $\eta(\mathbf{r}, t), \theta(\mathbf{r}, t)$ taken at the same point $\mathbf{r}$. 
In what follows we will find this conditions and obtain the corresponding particular form of expression (2.14)

3. Governing equations for the perfusion rate. General description. By virtue of the adopted assumptions the size $l_{N}$ of the elementary domains plays the role of an infinitesimal length. Therefore expression (2.4) can be rewritten as

$$
\eta(\mathbf{r})=\frac{1}{l_{N}^{d}} \sum_{i \in \mathbb{L}_{N}} J_{i} \Theta_{i}(\mathbf{r})
$$

where the symbol $\Theta_{i}(\mathbf{r})$ stands for the characteristic function of the fundamental domain $\mathfrak{M}_{i}$, i.e.

$$
\Theta_{i}(\mathbf{r})=\left\{\begin{array}{lll}
1 & \text { if } & \mathbf{r} \in \mathfrak{M}_{i} \\
0 & \text { if } & \mathbf{r} \notin \mathfrak{M}_{i}
\end{array}\right.
$$

and the sum runs over all the branches of the last level. If we find expressions relating the pattern $\left\{J_{i}\right\}$ to the fields $\theta(\mathbf{r}, t), \eta(\mathbf{r}, t)$ then formula (3.1) will immediately give us the specific form of (2.14). So in the present section we solve the equations stated above with respect to the variables $\left\{J_{i}\right\}$ regarding the field $\theta(\mathbf{r}, t)$ (and may be the field $\eta(\mathbf{r}, t))$ as fixed.

3.1. Kirchhoff's equations. Let us, first, find the extremal equations for functional (2.9). Following the Lagrange multiplier method we reduce the extremum problem for functional (2.9) subject to conditions (2.5) to finding the extremal equations of the following functional

$$
\mathbb{D}_{L}\left\{J_{i}\right\}=\left[\frac{1}{2} \sum_{i} R_{i} J_{i}^{2}-\mathcal{E}_{\text {ext }} J_{0}\right]+\sum_{\mathfrak{b}} P_{\mathfrak{b}}\left[\sum_{i \in \mathfrak{b}}^{\text {in }} J_{i}-\sum_{i \in \mathfrak{b}}^{\text {out }} J_{i}\right],
$$

where $\left\{P_{\mathfrak{b}}\right\}$ are the Lagrange multipliers ascribed to the nodes $\{\mathfrak{b}\}$ of the network $\mathfrak{N}$, the sum $\sum_{\mathfrak{b}}$ runs over all the nodes $\{\mathfrak{b}\}$, and the symbols $\sum_{i \in \mathfrak{b}}^{\text {in }} J_{i}, \sum_{i \in \mathfrak{b}}^{\text {out }} J_{i}$ stand for the sum over all the branches going in or out of the given node $\mathfrak{b}$. (The direction of the motion on the network $\mathfrak{N}$ is chosen so to coincide with the direction of transport agent flow.) From the conditions $\partial \mathbb{D}_{L} / \partial J_{i}=0$ we obtain the desired extremals

$$
J_{i} R_{i}=P_{\text {in }}^{i}-P_{\text {out }}^{i}
$$

and

$$
P_{\text {in }}^{0}-P_{\text {out }}^{0}=\mathcal{E}_{\text {ext }} .
$$

Here the multipliers $P_{\text {in }}^{i}, P_{\text {out }}^{i}$ correspond to the entrance and the exit of the given branch $i$ and $P_{\mathrm{in}}^{0}, P_{\mathrm{out}}^{0}$ are the multipliers ascribed to the entrance and the exit of the transport network. It should be noted that equations (3.4) and (3.5) together with equations (2.5) actually make up the system of Kirchhoff's equations for the network $\mathfrak{N}$. In these equations the variables $\left\{P_{\mathfrak{b}}\right\}$ play the role of potentials at the nodes $\{\mathfrak{b}\}$ causing the transport agent flow through the corresponding branches and the kinetic coefficients $\left\{R_{i}\right\}$ may be regarded as the branch resistances. 
3.2. Additional potential sources. At the next step we should solve the system of Kirchhoff's equations (2.5), (3.4), (3.5). However, when the field $\theta(\mathbf{r}, t)$ is nonuniform, the resistances $\left\{R_{i}\right\}$ of all the branches can differ in magnitude because of the network response. In this case solving Kirchhoff's equations directly is troublesome. In order to avoid this problem we make use of the following trick.

Let us ascribe to each branch $i$ the quantity $\varepsilon_{i}$ defined by the expression

$$
\varepsilon_{i}=J_{i}\left(R_{n_{i}}^{0}-R_{i}\right),
$$

where $n_{i}$ is the level number of the given branch. The quantities $\left\{\varepsilon_{i}\right\}$ will be called additional potential sources. Formula (3.6) allows us to rewrite equation (3.4) in terms of

$$
J_{i} R_{n_{i}}^{0}=P_{\text {in }}^{i}-P_{\text {out }}^{i}+\varepsilon_{i} .
$$

The collection of equations (2.5) and (3.7) written for every node and every branch forms the system of Kirchhoff's equations describing the flow pattern $\left\{J_{i}\right\}$ on a certain network of the same geometry where, however, branches do not respond to variations in $\left\{\theta_{i}\right\}$. In this case the self-regulation process is effectively implemented through the appearance of the additional potential sources $\left\{\varepsilon_{i}\right\}$. We will refer to this network as the homogeneous one. In this way, when the quantities $\left\{\varepsilon_{i}\right\}$ have known values the analysis of the transport agent flow redistribution over the supplying and draining beds with branches sensitive to $\theta_{i}$ is reduced to solving the Kirchhoff's equations for the corresponding homogeneous network. In the next subsection we will get expressions relating the additional potential sources $\left\{\varepsilon_{i}\right\}$ to the fields $\theta(\mathbf{r}, t), \eta(\mathbf{r}, t)$. Therefore this trick, in fact, can be useful in solving the original Kirchhoff's equations. In the present subsection we obtain the expressions specifying the flow pattern $\left\{J_{i}\right\}$ for fixed values of the quantities $\left\{\varepsilon_{i}\right\}$.

Since the flow patterns on the supplying and draining beds are mirror image of each other we may confine our consideration to the draining bed only. In this case we may set $P_{\text {out }}^{0}=0$ at the network exit and $P_{\text {in }}^{i}=\mathcal{E}_{\text {ext }} / 2$ for all the branches of the last level, $i \in \mathbb{L}_{N}$. For the corresponding draining bed of the homogeneous network the solution of Kirchhoff's equations (2.5), (3.7) can be written in the form

$$
J_{i}=\sum_{j} \Lambda_{i j} \varepsilon_{j}+\frac{1}{2} \Lambda_{i i_{0}} \mathcal{E}_{\text {ext }} .
$$

Here $\Lambda_{i j}$ is the Green matrix, i.e. the solution of these equations when $\mathcal{E}_{\text {ext }}=0$ and $\varepsilon_{j^{\prime}}=0$ for all branches except the branch $j$ for which $\varepsilon_{j}=1$, and $i_{0}$ stands for the draining bed stem. We note that the possibility of representation (3.8) results from the linearity of equations (2.5) and (3.7) with respect to the transport agent flows $\left\{J_{i}\right\}$. Appendix A specifies the Green matrix $\Lambda_{i j}$ at leading order in the small parameter $[\rho(n)-\rho(n+1)] / \rho(n)$ (for the definition of $\rho(n)$ see expression (2.11)).

Formula (3.8) enables us to represent relationship (3.1) in terms of

$$
\eta(\mathbf{r})=\frac{1}{l_{N}^{d}} \sum_{i \in \mathbb{L}_{N}}\left(\sum_{j} \Lambda_{i j} \varepsilon_{j}+\frac{1}{2} \Lambda_{i i_{0}} \mathcal{E}_{\mathrm{ext}}\right) \Theta_{i}(\mathbf{r}) .
$$

This expression will lead us to (2.14) if we can describe the dynamics of the additional potential sources $\left\{\varepsilon_{i}\right\}$. 
3.3. Governing equations for the additional potential sources. Differentiating relation (3.6) with respect to time $t$ and taking into account equation (2.12) we find the following evolution equation for the quantity $\varepsilon_{i}$ :

$$
\tau_{n_{i}} \frac{d \varepsilon_{i}}{d t}+\varepsilon_{i}\left[f\left(1-\frac{\varepsilon_{i}}{J_{i} R_{n_{i}}^{\circ}}\right)-\tau_{n_{i}} \frac{d}{d t} \ln J_{i}\right]=R_{n_{i}}^{0} J_{i} \frac{\theta_{i}}{\theta_{c}} .
$$

In obtaining (3.10) we also have assumed that the flow $J_{i}$ cannot change its direction. Due to the conservation laws (2.5) and (2.7) the values $J_{i}$ and $\theta_{i} J_{i}$ corresponding to the branch $i$ can be directly related to the same variables ascribed to the branches of the subtree $\mathfrak{N}^{i}$ whose stem is the given branch $i$. Passing to the last level $\mathbb{L}_{N}^{i}$ of this subtree we immediately get

$$
\begin{aligned}
J_{i} & =\sum_{j \in \mathbb{L}_{N}^{i}} J_{j}=\int_{\mathfrak{M}} d \mathbf{r} \Theta_{i}(\mathbf{r}) \eta(\mathbf{r}), \\
J_{i} \theta_{i} & =\sum_{j \in \mathbb{L}_{N}^{i}} J_{j} \theta_{j}=\int_{\mathfrak{M}} d \mathbf{r} \Theta_{i}(\mathbf{r}) \eta(\mathbf{r}) \theta(\mathbf{r}),
\end{aligned}
$$

where we have made us of expressions (2.4), (2.6) and the identity

$$
\sum_{j \in \mathbb{L}_{N}^{i}} \Theta_{j}(\mathbf{r})=\Theta_{i}(\mathbf{r})
$$

which is due to (2.2).

The system of equation (3.10) and expressions (3.11), (3.12) describes the dynamics of the additional potential sources in such a form that explicitly relates time variations of $\left\{\varepsilon_{i}\right\}$ to the fields $\theta(\mathbf{r}, t), \eta(\mathbf{r}, t)$ and, thus, among with expression (3.9) gives us, in principle, the desired relationship (2.14).

In particular, when the branches respond without delay, i.e. $\left\{\tau_{n}=0\right\}$, this system and expression (2.13) gives us the explicit relation

$$
\varepsilon_{i}(t)=R_{n_{i}}^{0}\left[\int_{\mathfrak{M}} d \mathbf{r} \eta(\mathbf{r}, t) \Theta_{i}(\mathbf{r})\right]\left[1-\phi\left(\frac{\int_{\mathfrak{M}} d \mathbf{r} \eta(\mathbf{r}, t) \theta(\mathbf{r}, t) \Theta_{i}(\mathbf{r})}{\int_{\mathfrak{M}} d \mathbf{r} \eta(\mathbf{r}, t) \Theta_{i}(\mathbf{r})}\right)\right] .
$$

The substitution of (3.14) into (3.9) directly specifies the functional dependence (2.14).

4. Ideal supplying network. In this section we show that there are special conditions under which the supplying network can function perfectly, namely, local variations in the concentration of life activity products $\theta(\mathbf{r}, t)$ cause variations in the perfusion rate $\eta(\mathbf{r}, t)$ of transport agent at the same point only. Besides, it turns out that under these conditions the response of the supplying network keeps the concentration of life activity products rigorously inside the vital interval. In other words, the field $\theta(\mathbf{r}, t)$ cannot go beyond the interval $\left(0, \theta_{c}\right)$ for a long time.

We consider a perturbation $\delta \theta(\mathbf{r}, t)$ of the field $\theta(\mathbf{r}, t)$ whose characteristic spatial scale $\mathcal{L}_{\theta}$ meets the inequality 


$$
\mathcal{L}_{\theta} \gg l_{N},
$$

so we may ignore nonuniformities of the field $\theta(\mathbf{r}, t)$ as well as the field $\eta(\mathbf{r}, t)$ on spatial scales about the elementary domain size $l_{N}$.

4.1. Linear model for branch response. Perfect self-regulation. Let us find the particular form of relationship (2.14) for the function $\phi(\theta)$ of the form

$$
\phi^{\text {id }}(\theta)=\left\{\begin{array}{clc}
1-\frac{\theta}{\theta_{c}}, & \text { if } & 0<\theta<\theta_{c} \\
0, & \text { if } & \theta>\theta_{c}
\end{array} .\right.
$$

This dependence $\phi^{\text {id }}(\theta)$ as well as the corresponding behavior of the function $f^{\text {id }}(x)$ is displayed in Fig. 2.2 by dotted lines. In particular, as follows from (2.13) $f^{\text {id }}(x)=1$ for $0<x \leq 1$ and $f^{\text {id }}(x)$ is undetermined at $x=0$.

Besides, we adopt the following additional assumptions. First, the delay time $\tau_{n}$ of the branch response is set equal for all the branches, i.e. $\tau_{n}=\tau$. Second, we will ignore the term $\tau \frac{d}{d t} \ln J_{i}$ in equation (3.10). This term actually describes dynamic interaction between additional potential sources and has no substantial effect at least in the following two limit cases. When $\tau \ll \tau_{\theta}$, where $\tau_{\theta}$ is the characteristic time of variations in the living medium state, the network response will be quasistationary, and the term $\tau \frac{d}{d t} \ln J_{i}$ along with the first one in (3.10) can be ignored. When $\tau \gg \tau_{\theta}$ and, in addition, the generation rate $q$ is not too high the value of $\varepsilon_{i} \tau \frac{d}{d t} \ln J_{i}$ should be negligibly small in comparison with $\tau \frac{d}{d t} \ln \varepsilon_{i}$. Indeed, in this case variations of the field $\theta(\mathbf{r}, t)$ are too fast for the supplying network response to follow them and the values of $\left\{\varepsilon_{j}\right\}$ should be small. Since time variations of the transport agent flow $J_{i}$ are due to the action of the additional potential sources $\left\{\varepsilon_{j}\right\}$ we may write $J_{i} \propto\left\{\varepsilon_{j}\right\}$ and, thus, the term $\varepsilon_{i} \tau \frac{d}{d t} \ln J_{i}$ is of second order in $\left\{\varepsilon_{j}\right\}$. Besides, the inequality $\varepsilon_{i}<J_{i} R_{n_{i}}^{0}$ is considered to be fulfilled in advance (below in the present section we will justify this assumption) and so $f^{\text {id }}\left(1-\varepsilon_{i} /\left(J_{i} R_{n_{i}}^{0}\right)\right)=1$.

Under these conditions we can rewrite equation (3.10) as

$$
\tau \frac{d \varepsilon_{i}}{d t}+\varepsilon_{i}=R_{n_{i}}^{0} \frac{1}{\theta_{c}} \int_{\mathfrak{M}} d \mathbf{r} \Theta_{i}(\mathbf{r}) \eta(\mathbf{r}) \theta(\mathbf{r}),
$$

where we have also taken into account relation (3.12). Then acting by the operator $\left(1+\tau \frac{d}{d t}\right)$ on equation (3.9) and using (4.3) we reduce it to the following

$$
\tau \frac{\partial \eta}{\partial t}+\eta=\eta_{0}+\frac{1}{\theta_{c}} \int_{\mathfrak{M}} d \mathbf{r}^{\prime} \sum_{j} \frac{1}{l_{N}^{d}} \sum_{i \in \mathfrak{L}_{N}} \Theta_{i}(\mathbf{r}) \Lambda_{i j} R_{n_{j}}^{0} \Theta_{j}\left(\mathbf{r}^{\prime}\right) \eta\left(\mathbf{r}^{\prime}\right) \theta\left(\mathbf{r}^{\prime}\right) .
$$

Here $\eta_{0}$ is the perfusion rate of transport agent through the living medium containing no life activity products, $\theta(\mathbf{r}, t)=0$. It should be pointed out that the value $\eta_{0}$ may be regarded as a certain phenomenological parameter in context of the self-regulation problem.

By virtue of inequality (4.1) we can simplify equation (4.4) in the following way. In the case under consideration there is a spatial scale $\ell^{*}$ such that $l_{N} \ll \ell^{*} \ll \mathcal{L}_{\theta}$ on 
which the fields $\eta(\mathbf{r}, t), \theta(\mathbf{r}, t)$ are practically constant. Let us choose a branch level $n^{*} \gg 1$ for which $l_{n^{*}} \approx \ell^{*}$ and $N-n^{*} \gg 1$. At the given level it is possible not to distinguish between the true perfusion rate $\eta(\mathbf{r}, t)$ and the perfusion rate $\eta^{*}(\mathbf{r}, t) \simeq$ $\eta(\mathbf{r}, t)$ averaged over the size $l_{n^{*}}$ of the fundamental domains $\mathbb{M}_{n^{*}}$. So we may specify this averaging, for example, in a manner as it is done in Appendix B, namely, $\eta^{*}(\mathbf{r}, t)=$ $\widehat{\mathcal{P}}_{n^{*}}\{\eta(\mathbf{r}, t)\}$. Then acting by the operator $\widehat{P}_{n^{*}}$ on equation (4.4) and taking into account identity $(\mathrm{B} .3)$ we convert it to the same form within the replacement

$$
\frac{1}{l_{N}^{d}} \sum_{i \in \mathbb{L}_{N}} \Theta_{i}(\mathbf{r}) \Lambda_{i j} \rightarrow \frac{1}{l_{n^{*}}^{d}} \sum_{i \in \mathbb{L}_{n^{*}}} \Theta_{i}(\mathbf{r}) \Lambda_{i j} .
$$

The latter, however, enables us to make use of identity (B.4) and to reduce (4.4) to

$$
\tau \frac{\partial \eta}{\partial t}+\eta=\eta_{0}+\frac{1}{\theta_{c}} \int_{\mathfrak{M}} d \mathbf{r}^{\prime} \frac{1}{l_{n^{*}}^{d}} \sum_{i \in \mathbb{L}_{n^{*}}} \Theta_{i}(\mathbf{r}) \Theta_{i}\left(\mathbf{r}^{\prime}\right) \eta\left(\mathbf{r}^{\prime}\right) \theta\left(\mathbf{r}^{\prime}\right) .
$$

Whence it immediately follows that

$$
\tau \frac{\partial \eta}{\partial t}+\left(1-\frac{\theta}{\theta_{c}}\right) \eta=\eta_{0}
$$

because the fields $\theta(\mathbf{r}, t), \eta(\mathbf{r}, t)$ are practically constant on scales of order $l_{n^{*}}$.

Equation (4.6) is the desired relationship between the perfusion rate $\eta(\mathbf{r}, t)$ of transport agent and the concentration $\theta(\mathbf{r}, t)$ of life activity products.

4.2. Characteristics of perfect self-regulation. The local form of equation (4.6) demonstrates that under the given conditions the perfusion rate $\eta(\mathbf{r}, t)$ is determined by variations in the field $\theta(\mathbf{r}, t)$ at the same point only. Thus, the cooperative response of all the branches is so self-consistent that the supplying network delivers "nutrients" only to the living medium points that "ask" for this. In this case no unexpected changes in the "nutrient" delivery interfering with the living medium activities occur, i.e. the supplying network functions perfectly.

Another characteristics of perfect self-regulation is the fact that the field $\theta(\mathbf{r}, t)$ cannot go beyond the vital interval $\left[0, \theta_{c}\right]$ for a long time. This is due to an unbounded increase in the perfusion rate $\eta(\mathbf{r}, t)$ that occurs when the field $\theta(\mathbf{r}, t)$ locally exceeds the value $\theta_{c}$ (see equation (4.6)). Typical dynamics of the fields $\theta(\mathbf{r}, t), \eta(\mathbf{r}, t)$ demonstrating such behavior is shown in Fig. 4.1 for the one-dimensional living medium.

Concluding this subsection we remind that when deriving equation (4.6) we have used the inequality $\varepsilon_{i}<J_{i} R_{n_{i}}^{0}$ without justification. Its validity can be shown in the following way. Let us consider the potential drop $\Delta P_{i} \equiv P_{\text {in }}^{i}-P_{\text {out }}^{i}$ across a branch $i$ which according to $(3.7)$ is equal to $\Delta P_{i}=J_{i} R_{n_{i}}^{0}-\varepsilon_{i}$. Then taking into account (3.11), (4.3), and (4.6) we get

$$
\tau \frac{d \Delta P_{i}}{d t}+\Delta P_{i}=R_{n_{i}}^{0} \eta_{0} \int_{\mathfrak{M}} d \mathbf{r} \Theta_{i}(\mathbf{r})
$$

The right-hand side of this equation is a constant independent of variations in the living medium state. At the initial time the field $\theta(\mathbf{r}, t)=0$ and so $\Delta P_{i}>0$, thus $\Delta P_{i}$ should be positive at any time moment later. 

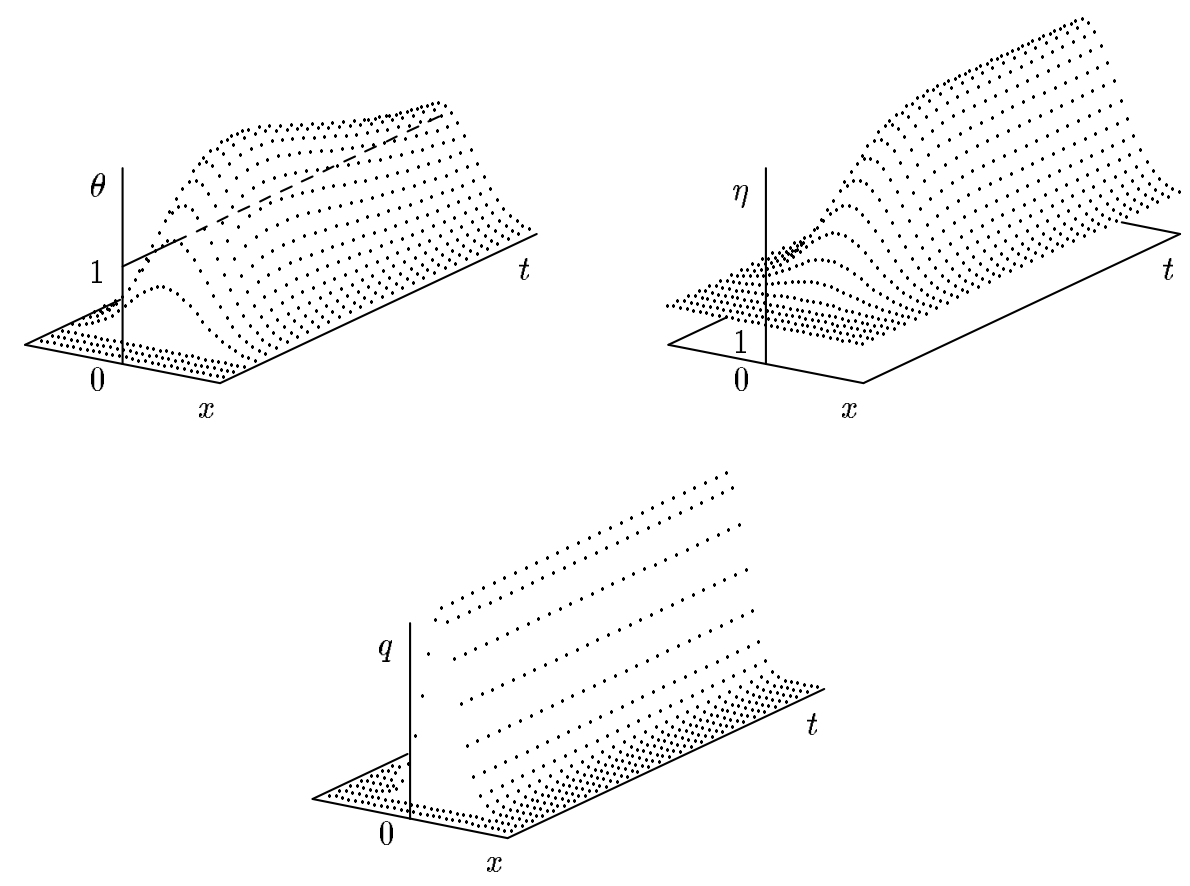

FIG. 4.1. Typical dynamics of the fields $\theta(\mathbf{r}, t), \eta(\mathbf{r}, t)$ under local stepwise variations in the life activities of the living medium $(q(\mathbf{r}, t)$ is the generation rate of life activity products).

Besides, equation (4.7) demonstrates one more characteristic property of the perfect self-regulation. Under the given conditions the potential distribution over the supplying network does not depend on variations in the living medium state. Exactly this independence ensures that the transport agent flow $J_{i}$ through a last level branch $i \in \mathbb{L}_{N}$ will remain unchanged if the state of the living medium inside the corresponding elementary domain $\mathfrak{M}_{i} \in \mathbb{M}_{N}$ does not alter. In fact, for this branch the value $\theta_{i} \cong \theta(\mathbf{r}, t)\left(\mathbf{r} \in \mathfrak{M}_{i}\right)$, so, the "resistivity" $R_{i}\left(\theta_{i}\right)$ of branch $i$ will be also constant under such conditions. Consequently, the transport agent flow $J_{i}=\Delta P_{i} / R_{i}\left(\theta_{i}\right)$ as well as the perfusion rate $\eta(\mathbf{r}, t)=J_{i} / l_{N}^{d}$ in this domain will be independent of the living medium state at other points. In other words, it is the constancy of the potential distribution that ensures the perfusion rate $\eta(\mathbf{r}, t)$ being controlled by the living medium state at the same point.

\section{Model generalizations.}

5.1. Dichotomic supplying network. In the previous sections we have considered the supplying network whose architectonics and embedding into the living medium are specified by $g$-fold spliting of branches with $g=2^{d}$, where $d$ is the living medium dimension. However, looking attentively through the previous analysis we see that it has been used, in fact, only the following properties of the supplying network architectonics. Each branch $i$ supplies (or drains) as a whole a certain domain $\mathfrak{M}^{i}$ and all the branches of smaller length and connected with the branch $i$ supply (or drain) subdomains of the domain $\mathfrak{M}^{i}$. The whole collection $\left\{\mathfrak{M}^{i}\right\}_{i \in \mathbb{L}_{n}}$ of the domains corresponding to branches $\left\{i \in \mathbb{L}_{n}\right\}$ of the same level $\mathbb{L}_{n}$ compose together the living medim $\mathfrak{M}$ and are mutually disjoint (see (2.1)). Besides, if $\left\{i \in \mathbb{L}_{n}^{j}\right\}$ is the whole 

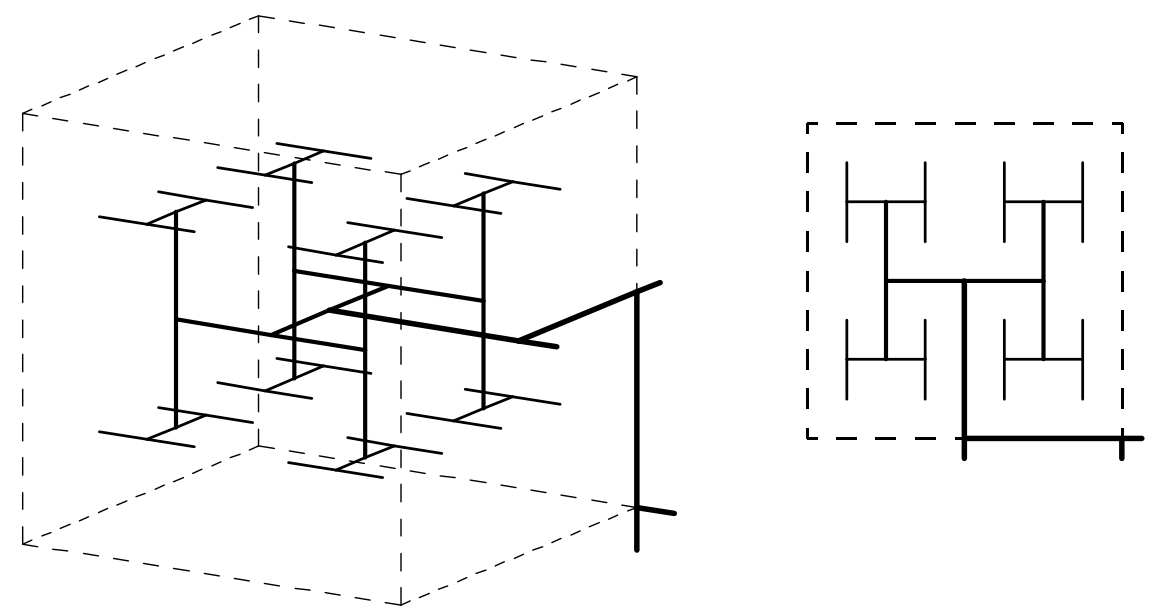

FIG. 5.1. Fragments of the supplying network architectonics for the two- and three-dimensional living medium.

collection of branches of level $n$ that are formed by splitting of the branch $j$ (of level $m \leq n$ ) then all the fundamental domains $\left\{\mathfrak{M}^{i}\right\}$ together make up the domain $\mathfrak{M}^{j}$ (see $(2.2)$ ). None of the other properties has been used.

In particular, the factor $g$ is not resticted by the value of $2^{d}$. Therefore the obtained results hold for any other implementation of the architectonics of the supplying network and its embedding into the living medium that meets conditioins (2.1), 2.2). For example, dichotomic supplying networks demonstrated in Fig. 5.1 obey these conditions.

5.2. Irregular supplying network. In the previous sections we have analyzed the self-regulation problem assuming the supplying network regular in architectonics and involving a large number of levelvs. So it could give the impression that these assumptions are the necessary conditions for self-regulation to be perfect. Therefore in the present section we demonstrate that the perfect self-regulation can occur in systems with supplying networks of arbitrary architectonics, at least, when the transient processes are ignorable.

As before we consider a supplying network consisting of supplying and draining beds which are mirror image of each other and connected through terminal branches (Fig. 5.2). The transport agent does not interact with the living medium until it reaches the terminal branches where the transport agent and the living medium exchange "nutrients" and life activity products. Then during motion through the draining bed the transport agent does not interact with the living medium again. Transport agent flow is assumed to be governed by the same regularities as stated in $\$ 2$.

So for each node $\mathfrak{b}$ of the draining bed (Fig. 5.3a) we can write the following conservation laws

$$
\sum_{i \in \mathfrak{b}}^{\text {in }} J_{i}=\sum_{i \in \mathfrak{b}}^{\text {out }} J_{i} \quad \text { and } \quad \sum_{i \in \mathfrak{b}}^{\text {in }} \theta_{i} J_{i}=\left(\theta_{\text {out }}\right)_{\mathfrak{b}} \sum_{i \in \mathfrak{b}}^{\text {out }} J_{i},
$$

where the sums $\sum_{i \in \mathfrak{b}}^{\text {in }}$ and $\sum_{i \in \mathfrak{b}}^{\text {out }}$ run over all the branches $i \in \mathfrak{b}$ leading to or from 


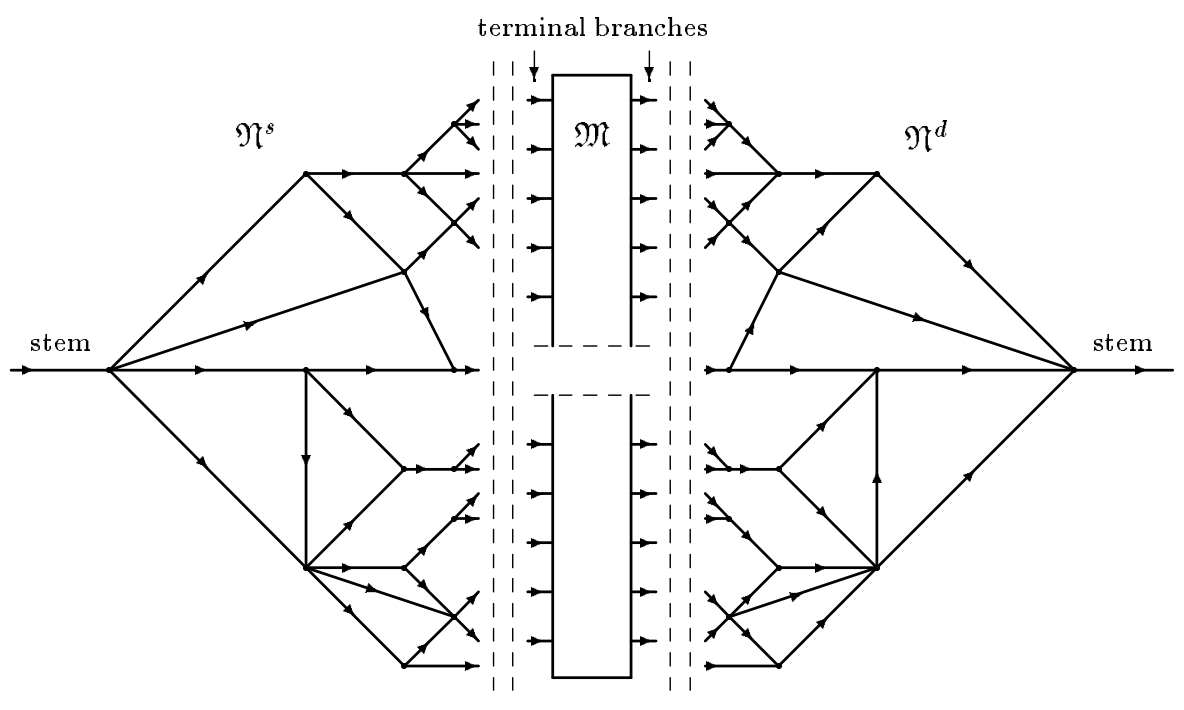

FIG. 5.2. Living system with irregular supplying network.

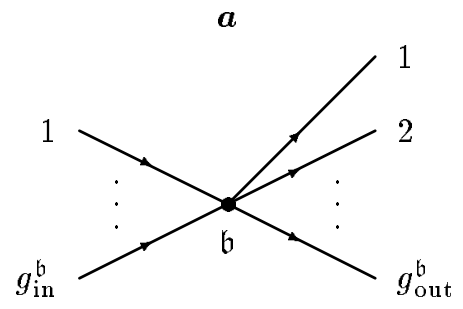

$\boldsymbol{b}$

FIG. 5.3. Elements of the irregular supplying network.

this node, $J_{i}$ and $\theta_{i}$ are the transport agent flow and the concentration of life activity products in the branche $i$ going in or out of the node $\mathfrak{b}$. Besides, for all the branches going out of one node $\mathfrak{b}$ the concentration $\left(\theta_{\text {out }}\right)_{\mathfrak{b}}$ of life activity products is assumed to be the same. The branch orientation is chosen according to the direction of the transport agent motion. The potential distribution $\left\{P_{\mathfrak{b}}\right\}$ over the draining bed nodes and the patten $\left\{J_{i}\right\}$ of transport agent flow are related by the expressions

$$
J_{i} R_{i}\left(\theta_{i}\right)=P_{\text {in }}^{i}-P_{\text {out }}^{i} \stackrel{\text { def }}{=} \Delta P_{i},
$$

where $P_{\text {in }}^{i}$ and $P_{\text {out }}^{i}$ are the potentials ascribed to the terminal nodes $\mathfrak{b}_{\text {in }}^{i}$ and $\mathfrak{b}_{\text {out }}^{i}$ of the branch $i$ as shown in Fig. 5.3 $\mathrm{b}$. The dependence of the "resistance" $R_{i}\left(\theta_{i}\right)$ of the branch $i$ on the value $\theta_{i}$ describes the living system response to variations in the living medium state. In the present section we analyze only the case corresponding to the perfect self-regulation which is characterized by the relationship (cf. (2.12))

$$
R_{i}\left(\theta_{i}\right)=\left\{\begin{array}{ccc}
R_{i}^{0}\left(1-\frac{\theta_{i}}{\theta_{c}}\right) & \text { if } & 0<\theta_{i} \leq 1 \\
0 & \text { if } & \theta_{i} \geq 1
\end{array}\right.
$$


where $R_{i}^{0}$ is the "resistance" of the branch $i$ at $\theta_{i}=0$. For the terminal branches $\{i\}^{\text {term }}$ of the draining bed the quantities $\left\{\theta_{i}\right\}^{\text {term }}$ are directly specified by the living medim state and, so, may be treated as predetermined when analyzing the distribution of transport agent flow over the draining bed. Besides, due to the mirror symmetry of the supplying and draining beds, we can regard the potential $P_{\text {out }}^{0}$ at the exit node of the draining bed (the latter node of the draining bed stem) and the potential $P_{\text {in }}^{\text {term }}$ at all the entrance nodes (the former nodes of the terminal branches) as fixed values.

In order to show that under such condition the supplying network response is perfect it is sufficient to demonstrate that the potential distribution over the draining bed nodes is constant, i.e. does not depends on variations in the field $\theta(\mathbf{r}, t)$. Indeed, in this case the perfusion rate $\eta(\mathbf{r}, t)$ of transport agent inside a domain $\mathfrak{M}_{i}^{\text {term }}$ drained as a whole by the terminal branch $i^{\text {term }}$ is

$$
\eta(\mathbf{r}, t) \simeq \frac{J_{i}^{\text {term }}}{V_{i}^{\text {term }}}=\frac{\Delta P_{i}^{\text {term }}}{V_{i}^{\text {term }} R_{i}\left(\theta_{i}^{\text {term }}\right)}=\frac{\text { const }}{R_{i}\left(\theta_{i}^{\text {term }}\right)}
$$

where $V_{i}^{\text {term }}$ is the volume of the domain $\mathfrak{M}_{i}^{\text {term }}$. Whence it follows that the perfusion rate $\eta(\mathbf{r}, t)$ is practically controlled by the field $\theta(\mathbf{r}, t)$ taken at the same point $\mathbf{r} \in \mathfrak{M}_{i}^{\text {term }}$ because the value of $\theta_{i}^{\text {term }}$ is determined directly by the value of the field $\theta(\mathbf{r}, t)$ in the domain $\mathfrak{M}_{i}^{\text {term }}$.

Let us fix a potential distribution $\left\{\tilde{P}_{\mathfrak{b}}\right\}$ that occurs at initial time. In general the system of Kirchhoff's equations (5.1), (5.2) should have a single solution. So to prove the independece of the potential distribution $\left\{P_{\mathfrak{b}}\right\}$ from variations in the field $\theta(\mathbf{r}, t)$ we may to show that this system possesses a solution provided we have fixed $\left\{P_{\mathfrak{b}}=\tilde{P}_{\mathfrak{b}}\right\}$.

Let us consider the system of equations (5.1), (5.2) for an arbitrary node $\mathfrak{b}$ (Fig. 5.3a) which is formed by $g_{\text {in }}^{\mathfrak{b}}$ branches going into it and $g_{\text {out }}^{\mathfrak{b}}$ branches leaving this node. At first, we will treat the values $\left\{\theta_{i}\right\}^{\text {in }}$ ascribed to the branches going into the node $\mathfrak{b}$ as given quantities. Then the variables to be found by solving this system are as follows: $\left\{J_{i}\right\}^{\text {in }},\left\{J_{i}\right\}^{\text {out }}$, and $\left(\theta_{\text {out }}\right)_{\mathfrak{b}}$ (the subsripts $i n$ and out mean that the corresponding quatities relate to the branches going into or out of the node $\mathfrak{b}$ ).

The total number of these quantities is equal to $g_{\text {in }}^{\mathfrak{b}}+g_{\text {out }}^{\mathfrak{b}}+1$, whereas the total number of the given equations is $g_{\text {in }}^{\mathfrak{b}}+g_{\text {out }}^{\mathfrak{b}}+2$. Therefore this system seems to be overdetermined. In fact, however, equations (5.1), (5.2) are linearly dependent.

To justify this statement, we, first, note that under the steady-state conditions none of the quantities $\left\{\theta_{i}\right\}$ can exceed the value $\theta_{c}$. Indeed, according to (5.1), for any node $\mathfrak{b}$ the value of $\left(\theta_{\text {out }}\right)_{\mathfrak{b}} \leq \max \left\{\theta_{i}\right\}_{\text {in }}$. So for a branch $i$ the value of $\theta_{i}$ might exceed $\theta_{c}$ only if there were a path $\mathbb{P}_{i}$ on the draining bed leading from the branch $i$ to one of the terminal branches such that for all braches $\{j\}$ belonging to it the values $\theta_{j} \geq \theta_{c}$. In this case, as follows from (5.2) and (5.3), the total "resistance" of the path $\mathbb{P}_{i}$ were equal to zero. So the potential $P_{\text {out }}^{i}$ at the node $\mathfrak{b}_{i}$ into which the branch $i$ goes would coinside with the potential at the draining bed entrance, $P_{\text {out }}^{i}=$ $P_{\text {in }}^{\text {term }}$. In addition, the total transport agent flow $\sum_{i \in \mathfrak{b}_{i}}^{\text {out }} J_{i}$ going through the node $\mathfrak{b}_{i}$ would be equal to the transport agent flow along the path $\mathbb{P}_{i}$ except for cases when there are another similar paths $\mathbb{P}_{i}^{\prime}$. This in turn would cause the value of $\left(\theta_{\text {out }}\right)_{\mathfrak{b}_{i}}$ to become greater than (or equal to) $\theta_{c}$ and so on. In this way we would reach the stem of the draining bed and obtain that the potential $P_{\text {out }}^{0}$ at the exit of the draining bed has to be equal to the portential $P_{\mathrm{in}}^{\text {term }}$ at its entrance. Thus all the values $\theta_{i}$ do not exceed $\theta_{c}$. 
Then let us sum equations (5.2) with the wight $1 / R_{i}^{0}$ over all branches relating to the node $\mathfrak{b}$. In this way taking into account (5.1) and (5.3) we get

$$
\sum_{i \in \mathfrak{b}}^{\text {in }} \frac{\tilde{P}_{\mathrm{in}}^{i}-\tilde{P}_{\mathfrak{b}}}{R_{i}^{0}}=\sum_{i \in \mathfrak{b}}^{\text {out }} \frac{\tilde{P}_{\mathfrak{b}}-\tilde{P}_{\text {out }}^{i}}{R_{i}^{0}} .
$$

Since the initial portential distribution $\left\{\tilde{P}_{\mathfrak{b}}\right\}$ has been established by the existing transport agent flow this equality is fulfilled. So equations (5.1), (5.2) are linearly dependent and can be reduced to a system of $\left(g_{\text {in }}^{\mathfrak{b}}+g_{\text {out }}^{\mathfrak{b}}+1\right)$ equations which is not overdetermined and possesses a solution.

Above we have regarded the quantities $\left\{\theta_{i}\right\}$ as given beforehand. If a node $\mathfrak{b}$ is one of the latter nodes of the terminal branches this is justified because the values $\left\{\theta_{i}\right\}$ at such branches are directly determined by the living medium state. Then, sovling the system of equations (5.1) $-(5.2)$ for these nodes we find the values $\left\{\left(\theta_{\text {out }}\right)_{\mathfrak{b}}\right\}$ which form the collection of quatities $\left\{\theta_{i}\right\}_{\text {int }}$ for nodes of lower levels. Repeating this proceedures we get the draining bed stem.

In this way we get the conclusion that there is a solution of the full set of equation (5.1)-(5.2) for a fixed potential distribution $\left\{P_{\mathfrak{b}}\right\}$ when the state of the living medium varies in time. The latter demonstrates that under the adopted assumptions the response of the supplying network is perfect.

6. Essence of perfect self-regulation. The fact that equation (4.6) governing the dynamics of transport agent perfusion can be of such a simple form is a surprise because it does not directly contain any information on the complex architectonics of the supplying network. This fact is actually one of the main results obtained in the present paper. So we, now, discuss the qualitative physical essence of haw the perfect regulation can occur.

Let us assume that in a small domain $\mathfrak{Q}$ (Fig. 6.1) the field $\theta(\mathbf{r}, t)$ increases and comes close to the boundary $\theta_{c}$ of the vital interval. In order to smother this dangerous growth the system should increase the perfusion rate $\eta(\mathbf{r}, t)$ in the domain $\mathfrak{Q}$. For this purpose all the "resistances" $\left\{R_{i}\right\}_{\mathbb{P}}$ along the path $\mathbb{P}$ connecting the stems of the supplying and draining beds and going through the domain $\mathfrak{Q}$ have to decrease substantially. As a result, the transport agent flow along the path $\mathbb{P}$ and, consequently, the perfusion rate in the domain $\mathfrak{Q}$ will grow. Higher values of the variables $\left\{\theta_{i}\right\}_{\mathbb{P}}$ along the path $\mathbb{P}$ (on the draining bed) bear the information required of such a response.

Variations in the field $\theta(\mathbf{r}, t)$ located in the domain $\mathfrak{Q}$ can, in principle, give rise to alterations of the perfusion rate at points external to the domain $\mathfrak{Q}$ because of variations in the transport agent flow through large branches. However there is a difference between the external and internal points of the domain $\mathfrak{Q}$ from the standpoint of this transport agent flow redistribution. For an external point $\mathbf{r} \notin \mathfrak{Q}$ on the path $\mathbb{P}$ there are two nodes $\mathfrak{b}_{\mathrm{r}}^{s}$ and $\mathfrak{b}_{\mathrm{r}}^{d}$ belonging to the supplying and draining beds, respectively, at which a similar path $\mathbb{P}_{\mathbf{r}}$ leading to the given point $\mathbf{r}$, at first, diverges from the path $\mathbb{P}$ and, then, they converge (Fig. 6.1). Due to the mirror symmetry of the two beds we can confine our consideration to the supplying bed only. The node $\mathfrak{b}_{\mathbf{r}}^{s}$ divides the path $\mathbb{P}$ on this bed into two parts. One of them leads from the stem to the node $b_{\mathbf{r}}^{s}$ and coincides with the path $\mathbb{P}_{\mathbf{r}}$. The other goes from the node $\mathfrak{b}_{\mathbf{r}}^{s}$ to the domain $\mathfrak{Q}$ and does not belong to the path $\mathbb{P}_{\mathbf{r}}$. Therefore, on one hand, decrease in the "resistances" of the branches forming the former part tends to increase the transport 


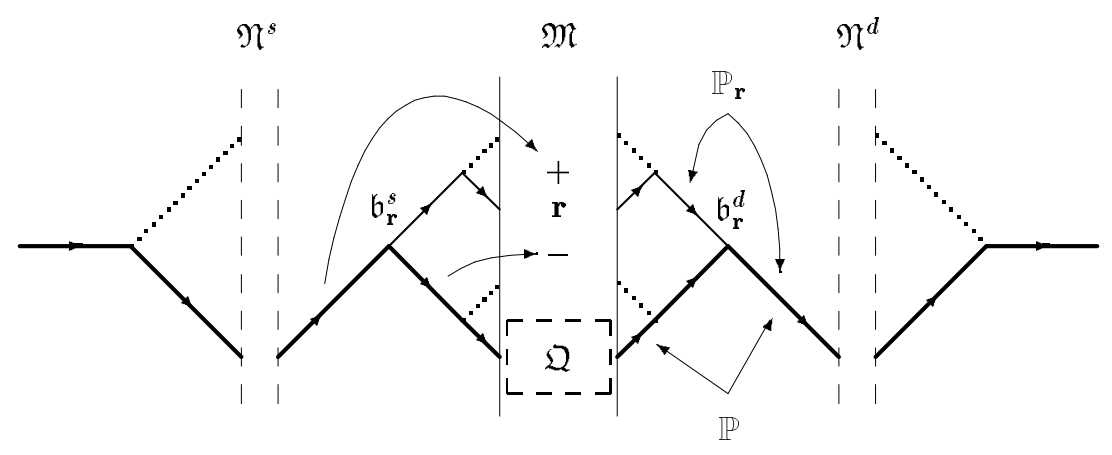

FiG. 6.1. Illustration of the mechanism by which the nonlocal dependence of the perfusion rate $\eta\{\theta\}$ on the field $\theta(\mathbf{r})$ at distant points is suppressed.

agent flow along the path $\mathbb{P}$ and, thus, through small branches of the path $\mathbb{P}_{\mathbf{r}}$ that directly control the perfusion rate in the vicinity of the point $\mathbf{r}$. On the other hand, the less are the "resistances" of the branches making up the latter part of the path $\mathbb{P}$, the larger is the fraction of transport agent flow that is directed along the path $\mathbb{P}$ at the node $\mathfrak{b}_{\mathbf{r}}^{s}$. These effects are opposite in sign with respect to increase in the perfusion rate at the point $\mathbf{r}$ as illustrated in Fig. 6.1 and can compensate each other. In the present paper we have actually found the conditions of such compensation.

7. Conclusion. In the present paper we have shown that there is a specific cooperative mechanism of self-regulation by which a living system can respond perfectly to changes in the environment, i.e. react without interference with itself. This mechanism is based on the corresponding self-processing of information and the cooperative effect caused by individual response of the supplying network elements. The existence of large hierarchical systems in nature and their capability for adapting to changes in the environment points to the fact that they should function perfectly, at least, at first approximation. For example, in living tissue in order to reduce effects of nonideality arterial and venous beds contain a system of anastomoses, i.e. vessels joining arteries or veins of the same level [1]. In other words, the vascular network is organized in such a manner that its function be as perfect as possible.

So we think the obtained results will be useful in analysis of real living systems and can form the basis of the following mathematical modelling of complex self-regulation processes.

Acknowledgements. The authors would like to thank Yu. A. Danilov and A. V. Priezzhev for attention to the work and useful criticisms.

\section{Appendix A. The Green matrix $\Lambda_{i j}$.}

By definition, the Green matrix $\Lambda_{i j}$ gives the flow pattern $\left\{J_{i}=\Lambda_{i j}: j\right.$ is fixed $\}$ on the homogeneous draining bed (Fig. A.1) provided the branch $j$ is fixed and contains a single potential source of power $\varepsilon_{j}=1$. (For simplicity of drawing we depict the binary draining bed in Fig. A.1.) In other words, as results from (2.5) and (3.7) the Green matrix $\Lambda_{i j}$ is the solution of the following system of the conservation equations at the nodes $\{\mathfrak{b}\}$ 


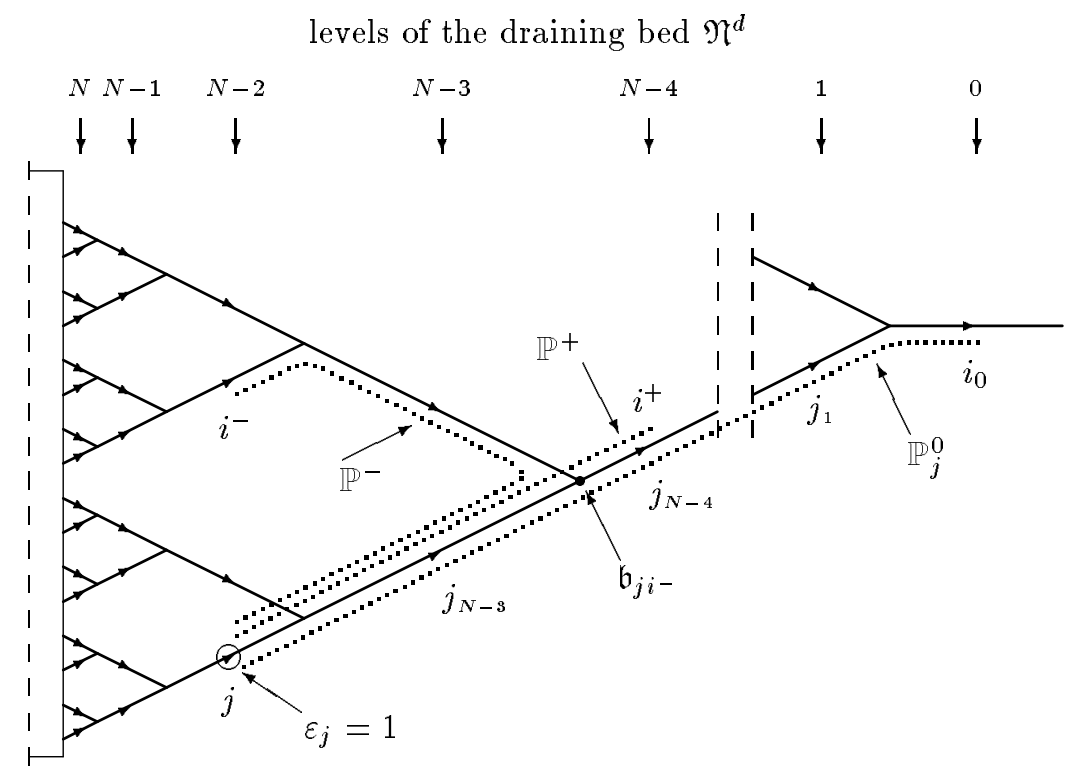

FIG. A.1. Homogeneous draining bed with a single potential source of unit power, $\varepsilon_{j}=1$, located at the branch $j$. (For simplicity is drawn the binary draining bed, $g=2$.)

$$
\sum_{i \in \mathfrak{b}}^{\text {in }} \Lambda_{i j}=\left(\Lambda_{i j}\right)_{\text {out }}^{\mathfrak{b}}
$$

and the potential equations

$$
\Lambda_{i j} R_{n_{i}}^{0}=P_{\mathrm{in}}^{i}-P_{\mathrm{out}}^{i}+\delta_{i j}
$$

Here the sum runs over all the branches $i \in \mathfrak{b}$ going into the node $\mathfrak{b}$ and $\delta_{i j}$ is the Kronecker symbol $\left(\delta_{i j}=1\right.$ if the branches $i, j$ coincide with each other and $\delta_{i j}=0$, otherwise).

In order to specify the Green matrix $\Lambda_{i j}$ we classify all the possible pairs of branches $\{i, j\}$ into two groups. The first group comprises all pairs $\{i, j\}_{+}$that can be joined by a path on the draining bed directed either from higher to lower levels or vice versa, i.e. by a path of constant direction. Such a path is depicted in Fig. A.1 by the dotted line $\mathbb{P}^{+}$. The second group involves the pairs $\{i, j\}$ - that can be joined by a path whose direction changes at a certain node $\mathfrak{b}_{i j}$ as shown in Fig. A.1 by the dotted curve $\mathbb{P}^{-}$. In other words, this path initially goes, for example, from the branch $j$ towards the stem until it reaches the node $\mathfrak{b}_{i j}$ (in Fig. A.1 it is the node $\left.\mathfrak{b}_{j i^{-}}\right)$and then goes towards the branch $i$ in the opposite direction. To the node $\mathfrak{b}_{i j}$ we also ascribe the level number $n_{i j}$ of branches going into it. The given classification allows us to state the following.

Proposition A.1. Let us introduce the quatities $\{Z(n)\}(n=0,1, \ldots, N)$ such that: 


$$
Z(n)=\sum_{m=n}^{N} \rho(m),
$$

where the function $\rho(m)$ is defined by formula (2.11). Then at leading order in the small parameter $\rho(n) / Z(n)$ the Green matrix $\Lambda_{i j}$ is specified by the expression for the $\{i, j\}+$ pairs

$$
\begin{array}{ll}
\Lambda_{i j}=\frac{1}{R_{0}} g^{-n_{j}} \frac{1}{Z\left(n_{i}\right)} \quad \text { if } \quad n_{i} \leq n_{j}, \\
\Lambda_{i j}=\frac{1}{R_{0}} g^{-n_{i}} \frac{1}{Z\left(n_{j}\right)} \quad \text { if } \quad n_{i} \geq n_{j},
\end{array}
$$

and for the $\{i, j\}$ - pairs

$$
\Lambda_{i j}=-\frac{1}{(g-1) R_{0}} g^{n_{i j}-n_{i}-n_{j}} \frac{\rho\left(n_{i j}\right)}{\left[Z\left(n_{i j}\right)\right]^{2}}
$$

where $n_{i}$ is the level number of branch $i$.

The given proposition is the main result of the present Appendix and the remaining part will be devoted to its substantiation.

Proof. Let us, first, prove this statement in the case when the branch $j$ is the stem $i_{0}$. The values of $\Lambda_{i i_{0}}$ are the same for all the branches $\left\{i: i \in \mathbb{L}_{n_{i}}\right\}$ belonging to one level $\mathbb{L}_{n_{i}}$. So from (A.1) we get

$$
\Lambda_{i i_{0}}=g^{-n_{i}} \Lambda_{i_{0} i_{0}}
$$

because level $\mathbb{L}_{n}$ involves exactly $g^{n}$ branches. Then choosing any path $\mathbb{P}$ on the draining bed leading form the stem $i_{0}$ to a branch $i_{N}$ of the last level and summing equations A.2 along this path we obtain

$$
\sum_{i \in \mathbb{P}} \Lambda_{i j} R_{n_{i}}^{0} \equiv \Lambda_{i_{0} i_{0}} R_{0} Z(0)=1,
$$

where we have taken into account expressions $(2.11)$ and (A.6). Whence we immediately find the expression for $\Lambda_{i_{0} i_{0}}$ and from (A.6) get the desired formula

$$
\Lambda_{i i_{0}}=g^{-n_{i}} \frac{1}{R_{0} Z(0)},
$$

which gives the same result as does formulae (A.4) (namely, (A.4b for $n_{i_{0}}=0$ ) because all the pairs $\left\{i i_{0}\right\}$ belong to the first group $\{i j\}_{+}$.

To find $\Lambda_{i j}$ for a branch $j$ whose level number $n_{j} \geq 1$ we consider a path $\mathbb{P}_{j}^{0}$ on the network shown in Fig. A.1 by the dotted line that goes from the given branch $j$ to the stem $i_{0}$. This path divides the draining bed into the path $\mathbb{P}_{j}^{0}$ itself and disjoint subtrees whose stems are connected with the path $\mathbb{P}_{j}^{0}$ through its nodes. The values of $\Lambda_{i j}$ for all branches of one level belonging to the same subtree are equal. This allows us to 

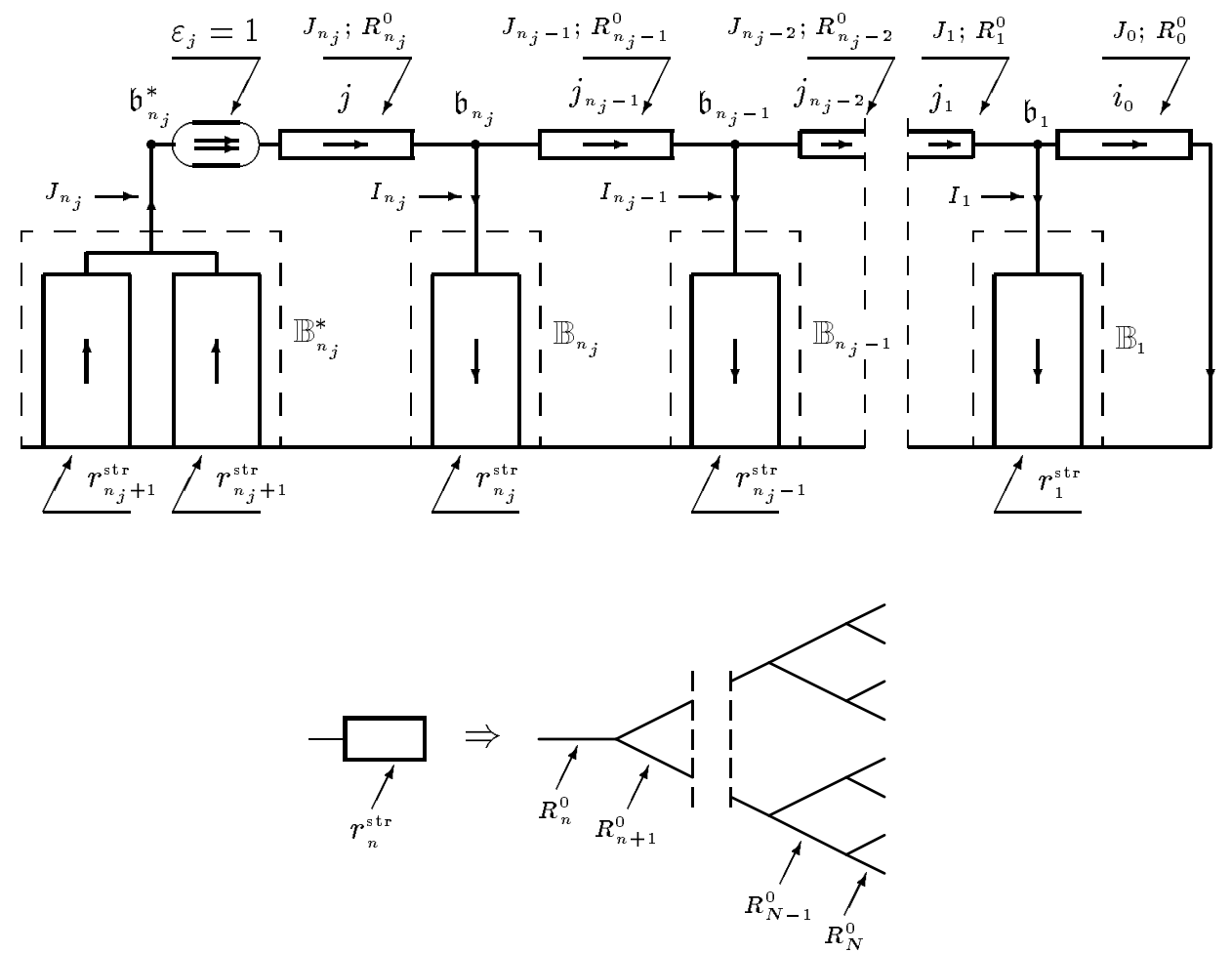

FIG. A.2. The block representation (upper graph) of the homogeneous draining bed equivalent to the graph shown in Fig. A.1 and the block structure (lower graph). (A block unites the draining bed subtrees characterized by identical transport agent flow distribution and $r_{n}^{\text {str }}$ is the total "resistance" of the corresponding subtree.)

transform the graph shown in Fig. A.1 into one shown in Fig. A.2, where the path $\mathbb{P}_{j}^{0}$ is represented by the sequence of branches designated by $\left\{j, j_{n_{j}-1}, j_{n_{j}-2}, \ldots, j_{1}, i_{0}\right\}$. Here the symbol $j_{n}$ stands for the branch of level $n$ belonging to this path, with $j_{n_{j}}$ and $j_{0}$ indicating the same as $j$ and $i_{0}$, respectively. The kinetic coefficients corresponding to these branches are $\left\{R_{n_{j}}^{0}, R_{n_{j}-1}^{0}, R_{n_{j}-2}^{0}, \ldots, R_{1}^{0}, R_{0}^{0}\right\}$. These branches at the terminal points (nodes bound up with their entrances and exits) $\left\{\mathfrak{b}_{n_{j}}^{*}, \mathfrak{b}_{n_{j}}, \mathfrak{b}_{n_{j}-1}, \ldots, \mathfrak{b}_{1}\right\}$ are connected with the blocks $\left\{\mathbb{B}_{n_{j}}^{*}, \mathbb{B}_{n_{j}}, \mathbb{B}_{n_{j}-1}, \ldots, \mathbb{B}_{1}\right\}$ of identical subtrees. These blocks except for the former one $\mathbb{B}_{n_{j}}^{*}$ (connected with the node $\left.\mathfrak{b}_{n_{j}}^{*}\right)$ involve $(g-1)$ subtrees, the block $\mathbb{B}_{n_{j}}^{*}$ contains $g$ subtrees. The transport agent flow spreads over such subtrees uniformly. Therefore each block, for example, $\mathbb{B}_{n}$ connected with a node $\mathfrak{b}_{n}$ can be treated as a single element characterized by the kinetic coefficient ("resistance")

$$
r_{n}=\frac{P_{\mathfrak{b}_{n}}}{I_{n}}
$$

Here $I_{n}$ is the total flow of transport agent through the given blook, $P_{\mathfrak{b}_{n}}$ is the potential at the node $\mathfrak{b}_{n}$ induced by the potential source $\varepsilon_{j}=1$, and for the sake of simplicity we have set the potential at the entrances of the last level branches equal to zero, $P_{\mathrm{in}}^{N}=0$. As is seen from the diagram shown in Fig. A.2 (lower graph), for $1 \leq n \leq n_{j}$ the value of $r_{n}$ is specified by the expression (see also Fig. A.2) 


$$
\begin{aligned}
r_{n} & =\frac{1}{(g-1)} r_{n}^{\operatorname{str}}=\frac{1}{g-1} R_{n}^{0}+\frac{1}{(g-1) g} R_{n+1}^{0}+\frac{1}{(g-1) g^{2}} R_{n+2}^{0}+\ldots \\
& =\frac{1}{(g-1)} \sum_{p=n}^{N} g^{-(p-n)} R_{p}^{0}
\end{aligned}
$$

because level $p \geq n$ contains $(g-1) g^{p-n}$ identical branches belonging to this block. Expressions (2.11) and (A.3) enable us to rewrite (A.10) as

$$
r_{n}=\frac{1}{(g-1)} g^{n} R_{0} Z(n) .
$$

In a similar way we obtain the expression for the kinetic coefficient of the last block connected with the path $\mathbb{P}_{j}^{0}$ through the node $\mathfrak{b}_{n_{j}}^{*}$

$$
r_{n_{j}+1}=g^{n_{j}} R_{0} Z\left(n_{j}+1\right) .
$$

Let us introduce the quantities

$$
J_{n} \stackrel{\text { def }}{=} \Lambda_{j_{n} j} \quad \text { and } \quad I_{n} \stackrel{\text { def }}{=} \sum_{j_{\text {stem }} \in \mathfrak{b}_{n}} \Lambda_{j_{\text {stem }} j}
$$

being the transport agent flow through a branch $j_{n}$ and the total transport agent flow in the subtree block $\mathbb{B}_{n}$, respectively, which are induced by the potential source $\varepsilon_{j}=1$. In the last expression the sum runs over all the stems of the subtrees forming the block $\mathbb{B}_{n}$. Then the given reduction of the initial graph to the subtree blocks (Fig. A.2 enables us to rewrite the Kirchhoff equations (A.1), A.2) in the form for $1 \leq n \leq n_{j}$ :

$$
I_{n}=J_{n}-J_{n-1}
$$

and for $2 \leq n \leq n_{j}$

$$
I_{n} r_{n}=J_{n-1} R_{n-1}^{0}+I_{n-1} r_{n-1} .
$$

These equations are completed by the following expressions bound up with the first and the last elements of the given network:

$$
\begin{gathered}
I_{1} r_{1}=J_{0} R_{0}, \\
J_{n_{j}}\left[r_{n_{j}+1}+R_{n_{j}}^{0}\right]+I_{n_{j}} r_{n_{j}}=1 .
\end{gathered}
$$

Due to $\rho(n)$ being a smooth function of $n$ the ratio $\rho(n) / Z(n)$ may be treated as a small value for $N-n \gg 1$. The latter allows us to find directly the solution of equations A.13 - A.16), which is matter of the next step. 
Continuous solution of equations ( $(\mathbf{A . 1 3})-(\mathbf{A . 1 6})$ Taking into account (2.11), (A.3), (A.11), and A.12 we can rewrite equation (A.14)-(A.16) in terms of

$$
g I_{n} Z(n)=(g-1) J_{n-1} \rho(n-1)+I_{n-1} Z(n-1)
$$

for $2 \leq n \leq n_{j}$ and

$$
\begin{gathered}
g I_{1} Z(1)=(g-1) J_{0}, \\
J_{n_{j}}+\frac{1}{(g-1)} I_{n_{j}}=\frac{1}{R_{0} Z\left(n_{j}\right)} g^{-n_{j}},
\end{gathered}
$$

where, in addition, we have used the identity $Z(n+1)+\rho(n)=Z(n)$ and the equality $\rho(0)=1$.

Let us analyze, at first, the solution of equations (A.13) and (A.17) when $n \sim 1$. For such values of $n$ the function $\rho(n)$ as well as $Z(n)$ may be considered as being constant, $\rho(n) \cong \rho(0)=1$ and $Z(n) \cong Z(0) \gg 1$ due to smoothness of the function $\rho(n)$. We seek the solution of these equations in the form

$$
J_{n}=A_{+} \zeta_{+}^{n}+A_{-} \zeta_{-}^{n},
$$

where $A_{+,-} ; \zeta_{+,-}$are some constants. Substituting A.20 into (A.13) we get

$$
I_{n}=A_{+} \zeta_{+}^{(n-1)}\left(\zeta_{+}-1\right)+A_{-} \zeta_{-}^{(n-1)}\left(\zeta_{-}-1\right)
$$

Then the substitution of this expression into (A.17) shows us that the constants $\zeta_{+}, \zeta_{-}$ are the roots of the equation

$$
(\zeta-1)(g \zeta-1)=\frac{g-1}{Z(0)} \zeta .
$$

Whence it follows that to the first order in the small parameter $1 / Z(0)$ :

$$
\zeta_{+}=1+\frac{1}{Z(0)}, \quad \zeta_{-}=\frac{1}{g}\left(1-\frac{1}{Z(0)}\right) .
$$

To the same order in $1 / Z(0)$ from $(\mathrm{A.13})$ and $(\mathrm{A.18})$ we find that the constants $A_{+}, A_{-}$ are related by the expression

$$
\frac{A_{-}}{A_{+}}=\frac{1}{(g-1) Z(0)} \ll 1 .
$$

According to $(\mathrm{A} .23)$ the first terms on the right-hand side of $(\mathrm{A} .2 \mathrm{C})$ and $(\mathrm{A} .21)$ are increasing functions of $n$, whereas the second ones are decreasing functions. Therefore, by virtue of (A.24) in the two expressions the former term is substantially greater than the latter for all $n \sim 1$. So, we may ignore the second term and regard the first one 
as a function of the continuous variable $n$ because of $\left(\zeta_{+}-1\right) \ll 1$. This allows us to set

$$
J_{n}-J_{n-1}=\frac{\partial J_{n}}{\partial n}
$$

in equation (A.13) and $I_{n-1} \approx I_{n}$ in $(\mathrm{A.17})$, and also to rewrite $(\mathrm{A} .20)$ in terms of

$$
J_{n} \simeq A_{+} \exp \left\{\frac{n}{Z(0)}\right\} .
$$

In this case the system of equations $(\mathrm{A.13})$ and $\mathrm{A.17}$ ) is reduced to the equation

$$
\frac{\partial J_{n}}{\partial n}=\frac{1}{Z(0)} J_{n}
$$

and function A.27) is its general solution. So, the "boundary condition" (A.18) is responsible only for the existence of the second terms in (A.20), (A.21) and, therefore, can be ignored.

As follows from equations (A.13), (A.14) (or (A.17) ) their general solution contains two arbitrary constants, for example, $J_{0}, J_{1}$. Indeed, all the other values of $I_{n}$ and $J_{n}$ can be found by iteration. The same situation is met with respect to the general solution (A.20), (A.21) of this equations for $n \sim 1$. So there is no solution of equations A.13), (A.14) different from (A.20), (A.21) for such values of $n$. Thereby, the influence of the "boundary condition" (A.15) on the solution of equations (A.13) and (A.14) is ignorable and we may seek this solution in the class of smooth functions of the continuous variable $n$. In this case it contains only one arbitrary constant specified by the "boundary condition" A.16) (or A.19)).

Within the framework of the adopted assumptions the quantities $J_{n}$ and $I_{n}$ as well as $\rho(n)$ and $Z(n)$ are related by the expressions

$$
\begin{gathered}
I_{n}=\frac{\partial J_{n}}{\partial n}, \\
\rho_{n}=-\frac{\partial Z(n)}{\partial n},
\end{gathered}
$$

and the system of equations (A.13), (A.17), (A.19) is reduces to the following equation

$$
\frac{\partial J_{n}}{\partial n} Z(n)+J_{n} \frac{\partial Z(n)}{\partial n}=0
$$

subject to the boundary condition

$$
J_{n_{j}}=g^{-n_{j}} \frac{1}{R_{0} Z\left(n_{j}\right)} .
$$

In obtaining these expressions we have also taken into account relation (A.13) and the inequalities $\left|I_{n+1}-I_{n}\right| \ll I_{n}$ and $|Z(n+1)-Z(n)| \ll Z(n)$. Besides, we have 
ignored the second term on the left-hand side of (A.19) because of $I_{n_{j}}=J_{n_{j}-1}-J_{n_{j}} \ll$ $J_{n_{j}}$. The solution of equation A.30 meeting the boundary condition A.31) is of the form

$$
J_{n}=g^{-n_{j}} \frac{1}{R_{0} Z(n)}
$$

and, thus, from $\mathrm{A.28}$ ) and (A.29) we obtain

$$
I_{n}=g^{-n_{j}} \frac{\rho(n)}{R_{0}[Z(n)]^{2}} .
$$

For a branch $i$ located on the path $\mathbb{P}_{j}^{0}$, i.e. $i \in\left\{j, j_{n_{j}-1}, j_{n_{j}-2}, \ldots, j_{1}, i_{0}\right\}$ the pair $\{i j\}$ belongs to the first group and $\Lambda_{i j}=J_{n_{i}}\left(n_{i}\right.$ is the level number of the branch $i$ ). Whence we directly get formula A.4a). If a branch $i$ belongs to the last block $\mathbb{B}_{n_{j}}^{*}$ of subtrees it level number $n_{i}<n_{j}$ and the pair $\{i j\}$ also belongs to the first group. Since for a fixed value of $n_{i}$ the total number of such branches is $g^{n_{i}-n_{j}}$ and the transport agent flow $\left(\Lambda_{i j}\right)$ is the same for all of them, we can write $\Lambda_{i j}=J_{n_{j}} / g^{n_{i}-n_{j}}$, which together with expression A.32 gives equality (A.4b).

Now let us consider a branch $i$ located in one of the blocks $\left\{\mathbb{B}_{n_{j}}, \mathbb{B}_{n_{j}-1}, \ldots, \mathbb{B}_{1}\right\}$, e.g. in a block $\mathbb{B}_{n_{\mathfrak{b}}}$. For this branch the pair $\{i j\}$ belongs to the second group and the node $\mathfrak{b}$ at which the block $\mathbb{B}_{n_{\mathfrak{b}}}$ joins the path $\mathbb{P}_{j}^{0}$ plays the role of the node $\mathfrak{b}_{i j}$ of the pair $\{i j\}_{-}$. For a fixed level $n_{i} \geq n_{\mathfrak{b}}$ the total number of such branches is $(g-1) g^{n_{i}-n_{\mathfrak{b}}}$ and the total transport agent flow going through this block is $I_{n_{\mathfrak{b}}}$. So in this case $\Lambda_{i j}=-I_{n_{B}} /\left[(g-1) g^{n_{i}-n_{\mathfrak{b}}}\right]$, whence taking into account A.33) we get formula (A.5). In this expression the sign "-" means that in the given branch $i$ the transport agent flow $\Lambda_{i j}$ caused by the additional potential source $\varepsilon_{j}=1$ is directed from lower to higher levels. In other words, this component of the transport agent flow through the branch $i$ and the total transport agent flow $J_{i}$ induced by the collective action of all the potential sources have opposite directions.

In this way we have considered all the branches, so, proven the stated proposition.

\section{Appendix B. The embedding identities.}

Let us specify the averaging procedure $\hat{\mathcal{P}}_{n}: \Psi(\mathbf{r}) \rightarrow \tilde{\Psi}(\mathbf{r})$ of a function $\Psi(\mathbf{r})$ over spatial scales of the fundamental domains $\left\{\mathfrak{M}_{n}\right\}$ of level $n$ by the expression

$$
\widehat{P}_{n}\{\Psi(\mathbf{r})\} \stackrel{\text { def }}{=} \int_{\mathfrak{M}} d \mathbf{r}^{\prime} P_{n}\left(\mathbf{r}, \mathbf{r}^{\prime}\right) \Psi\left(\mathbf{r}^{\prime}\right),
$$

where the kernel

$$
P_{n}\left(\mathbf{r}, \mathbf{r}^{\prime}\right)=\frac{1}{l_{n}^{d}} \sum_{i \in \mathbb{L}_{n}} \Theta_{i}(\mathbf{r}) \Theta_{i}\left(\mathbf{r}^{\prime}\right)
$$

and the sum runs over all the branches $\{i\}$ forming the level $\mathbb{L}_{n}$, for example, of the draining bed. Then we can state the followig.

Proposition B.1. Let $\mathbb{L}_{n}$ and $\mathbb{L}_{m}$ be the branch collections forming the levels $n$ and $m$, respectively, and $n \geq m$. Then 


$$
\frac{1}{l_{m}^{d}} \sum_{i \in \mathbb{L}_{m}} \Theta_{i}(\mathbf{r}) \Lambda_{i j}=\widehat{P}_{m}\left\{\frac{1}{l_{n}^{d}} \sum_{i \in \mathbb{L}_{n}} \Theta_{i}(\mathbf{r}) \Lambda_{i j}\right\} .
$$

Proof. We prove identity (B.3) by the direct substitution of $(\overline{\mathrm{B} .2})$ into the righthand side of (B.3). In this way we get

$$
\begin{aligned}
\widehat{P}_{m}\left\{\frac{1}{l_{n}^{d}} \sum_{i \in \mathbb{L}_{n}} \Theta_{i}(\mathbf{r}) \Lambda_{i j}\right\} & =\frac{1}{l_{m}^{d}} \sum_{i \in \mathbb{L}_{m}} \sum_{i^{\prime} \in \mathbb{L}_{n}} \Theta_{i}(\mathbf{r}) \Lambda_{i^{\prime} j} \frac{1}{l_{n}^{d}} \int_{\mathfrak{M}} d \mathbf{r}^{\prime} \Theta_{i}\left(\mathbf{r}^{\prime}\right) \Theta_{i^{\prime}}\left(\mathbf{r}^{\prime}\right) \\
& =\frac{1}{l_{m}^{d}} \sum_{i \in \mathbb{L}_{m}} \Theta_{i}(\mathbf{r}) \sum_{i^{\prime} \in \mathbb{L}_{n}^{i}} \Lambda_{i^{\prime} j}=\frac{1}{l_{m}^{d}} \sum_{i \in \mathbb{L}_{m}} \Theta_{i}(\mathbf{r}) \Lambda_{i j} .
\end{aligned}
$$

Here we have taken into account that, first,

$$
\frac{1}{l_{n}^{d}} \int_{\mathfrak{M}} d \mathbf{r}^{\prime} \Theta_{i}\left(\mathbf{r}^{\prime}\right) \Theta_{i^{\prime}}\left(\mathbf{r}^{\prime}\right)=\left\{\begin{array}{lll}
1, & \text { if } & i^{\prime} \in \mathbb{L}_{n}^{i} \\
0, & \text { if } \quad i^{\prime} \notin \mathbb{L}_{n}^{i}
\end{array},\right.
$$

where $\mathbb{L}_{n}^{i}$ is the full collection $\left\{i^{\prime}\right\}$ of branches of level $\mathbb{L}_{n}$ belonging to the subtree whose stem is the branch $i$ and, thus, $\mathfrak{M}_{i^{\prime}} \subset \mathfrak{M}_{i}$, and, second,

$$
\Lambda_{i j}=\sum_{i^{\prime} \in \mathbb{L}_{n}^{i}} \Lambda_{i^{\prime} j}
$$

because of the conservation laws A.1.

In the present paper we consider the model for the supplying network which envolves large but fininte number of hierarchy levels. In other words, we regard the total number $N$ of levels as a fixed (but large) value. In this case we can state the following.

Proposition B.2. At leading order in the small parameter $\rho\left(n_{i}\right) / Z\left(n_{i}\right)$ for almost all points $\mathbf{r} \in \mathfrak{M}$ it is fulfilled the identity

$$
\sum_{j \in \mathfrak{N}^{d}} \Lambda_{i j} R_{n_{j}}^{0} \Theta_{j}(\mathbf{r})=\Theta_{i}(\mathbf{r}),
$$

provided the level number $n_{i}$ of the branch $i$ meets the inequality $1 \ll n_{i} \ll N$.

In this context the term "leading order" means, in addition, that in (B.4) we ignore any summand caused by variations of the quantities $\rho(n)$ and $Z(n)$ when the argument $n$ changes on scale of order unity. This is due to such summands cannot be analyzed in the framework of the adopted continuous approximation of Kirchhoff's equations.

Let us prove this assertion.

Proof. For a finite number of hierarchy levels the volumentric measure of the interface made up of the boundaries of all the fundamental domains is equal to zero. So in proving (B.4) we may ignore all the points belonging to this interface. Therefore 


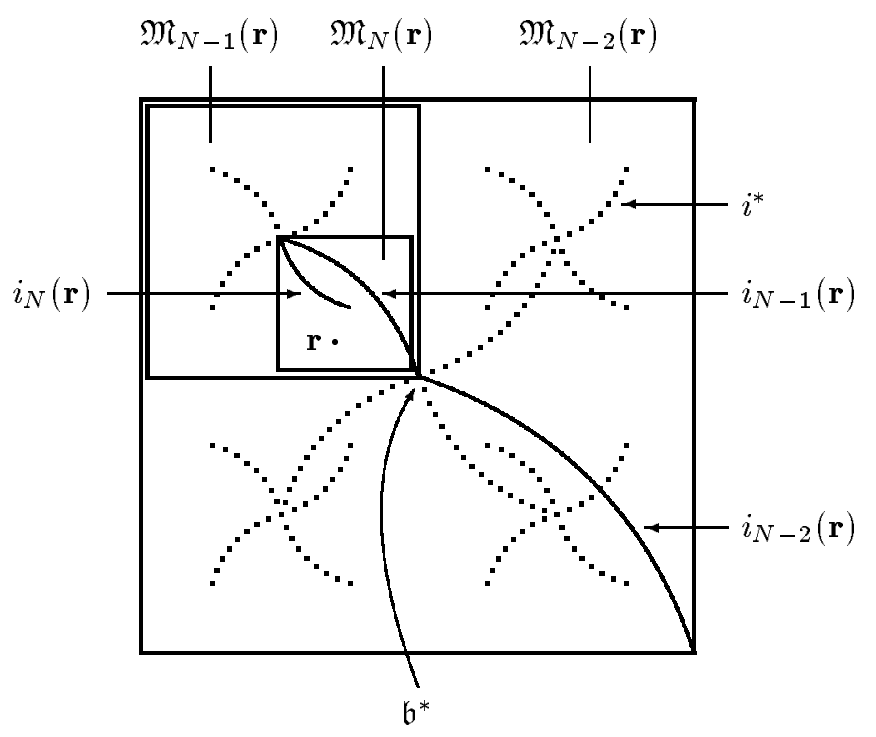

FIG. B.1. Illustration of constructing the domain collection $\left\{\mathfrak{M}_{n}(\mathbf{r})\right\}$ and the collection of the corresponding branches $\left\{i_{n}(\mathbf{r})\right\}$ forming the path $\mathbb{P}(\mathbf{r})$ (solid line) on the draining bed (dotted lines). (For simplicity is shown the two-dimensional living medium.)

let $\mathbf{r}$ be a point of the living medium domain, $\mathbf{r} \in \mathfrak{M}$, that does not belong to the boundary of any fundamental domain. For each level number $n$ this point $\mathbf{r}$ is located inside just one fundamental domain of level $n$ which will be labelled as $\mathfrak{M}_{n}(\mathbf{r})$. In this way we can build up the collection $\left\{\mathfrak{M}_{n}(\mathbf{r})\right\}(n=0,1, \ldots, N)$ of all the fundamental domains containing the point $\mathbf{r}$ (Fig. B.1). These domains in turn specify the sequence $\left\{i_{n}(\mathbf{r})\right\}=\left\{i_{o}, i_{1}(\mathbf{r}), i_{2}(\mathbf{r}), \ldots, i_{N}(\mathbf{r})\right\}$ of connected branches whose $n$-th term is the branch $i_{n}(\mathbf{r})$ of level $n$ contained in the domain $\mathfrak{M}_{n}(\mathbf{r})$. The branches $\left\{i_{n}(\mathbf{r})\right\}$ form a continuous path $\mathbb{P}(\mathbf{r})$ on the draining bed $\mathfrak{N}^{d}$ that leads from the stem $i_{0}$ to the branch $i_{N}(\mathbf{r})$ of the last level.

In formula (B.4) the functions $\left\{\Theta_{j}(\mathbf{r})\right\}$ differ from zero for the branches $\left\{i_{n}(\mathbf{r})\right\}$ only, thus

$$
\tilde{\Lambda}_{i}(\mathbf{r}) \stackrel{\text { def }}{=} \sum_{j \in \mathfrak{N}_{d}} \Lambda_{i j} R_{n_{j}}^{0} \Theta_{j}(\mathbf{r})=\sum_{j \in \mathbb{P}(\mathbf{r})} \Lambda_{i j} R_{n_{j}}^{0}
$$

Let us, first, consider a branch $i$ being one of the branches $\left\{i_{n}(\mathbf{r})\right\}$ whose level number $n_{i}$ meets the inequality $N-n_{i} \gg 1$. For the given branch all the pairs $\left\{i, i_{n}(\mathbf{r})\right\}$ belong to the first group specified in Appendix A. Then taking into account expressions (2.11), (A.3), and (A.4) we can rewrite (B.5) as

$$
\begin{aligned}
\tilde{\Lambda}_{i}(\mathbf{r}) & =\sum_{j \in \mathbb{P}(\mathbf{r})}^{n_{j} \geq n_{i}} \Lambda_{i j} R_{n_{j}}^{0}+\sum_{j \in \mathbb{P}(\mathbf{r})}^{n_{j}<n_{i}} \Lambda_{i j} R_{n_{j}}^{0} \\
& =\frac{1}{Z\left(n_{i}\right)} \sum_{n_{j}=n_{i}}^{N} \rho\left(n_{j}\right)+\sum_{n_{j}=0}^{n_{i}-1} g^{-\left(n_{i}-n_{j}\right)} \frac{\rho\left(n_{j}\right)}{Z\left(n_{j}\right)}
\end{aligned}
$$


and, thus,

$$
\tilde{\Lambda}_{i}(\mathbf{r})=1+\sum_{p=1}^{n_{i}} g^{-p} \frac{\rho\left(n_{i}-p\right)}{Z\left(n_{i}-p\right)} \cong 1
$$

at leading order in the small parameter $\rho\left(n_{i}\right) / Z\left(n_{i}\right)$ for $N-n_{i} \gg 1$.

Let us, now, consider a branch $i$ not belonging to the branch collection $\left\{i_{n}(\mathbf{r})\right\}$ (in Fig. B.1 it is the branch $i^{*}$ ). In this case there are a single node $\mathfrak{b}$ (node $\mathfrak{b}^{*}$ in Fig. B.1) on the path $\mathbb{P}(\mathbf{r})$ and a single subtree that contains the branch $i$ and whose stem joins the path path $\mathbb{P}(\mathbf{r})$ through the node $\mathfrak{b}$. The node $\mathfrak{b}$ devides the path $\mathbb{P}(\mathbf{r})$ into two parts, one going from the last level branch $i_{N}(\mathbf{r})$ to the node $\mathfrak{b}$ and the other leading from the node $\mathfrak{b}$ to the stem of the draining bed. Let us ascribe to the node $\mathfrak{b}$ the level number $n_{\mathfrak{b}}$ of branches going into it. Then we can say that the former

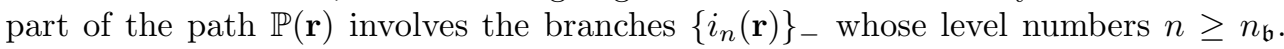
For such branches the pairs $\left\{i, i_{n}(\mathbf{r})\right\}_{-}$belong to the second group (see Appendix A and $n_{\mathfrak{b}}=n_{i i_{n}(\mathbf{r})}$. The latter part is made up of the branches $\left\{i_{n}(\mathbf{r})\right\}_{+}$whose level numbers $n<n_{\mathfrak{b}}$ and for these branches the pairs $\left\{i, i_{n}(\mathbf{r})\right\}_{+}$belong to the first group. In the given case taking into account expressions (2.11), (A.3), (A.4), and (A.5) we can rewrite $(\overline{B .5})$ as

$$
\begin{aligned}
\tilde{\Lambda}_{i}(\mathbf{r}) & =\sum_{j \in \mathbb{P}(\mathbf{r})}^{n_{j} \geq n_{\mathfrak{b}}} \Lambda_{i j} R_{n_{j}}^{0}+\sum_{j \in \mathbb{P}(\mathbf{r})}^{n_{j}<n_{\mathfrak{b}}} \Lambda_{i j} R_{n_{j}}^{0} \\
& =-\frac{1}{(g-1)} g^{n_{\mathfrak{b}}-n_{\mathfrak{i}}} \frac{\rho\left(n_{\mathfrak{b}}\right)}{\left[Z\left(n_{\mathfrak{b}}\right)\right]^{2}} \sum_{n_{j} \geq n_{\mathfrak{b}}} \rho\left(n_{j}\right)+\sum_{n_{j}<n_{\mathfrak{b}}} g^{n_{j}-n_{i}} \frac{\rho\left(n_{j}\right)}{Z\left(n_{j}\right)} \\
& =-\frac{1}{(g-1)} g^{n_{\mathfrak{b}}-n_{i}} \frac{\rho\left(n_{\mathfrak{b}}\right)}{Z\left(n_{\mathfrak{b}}\right)}+g^{n_{\mathfrak{b}}-n_{i}} \sum_{p=1}^{n_{\mathfrak{b}}} g^{-p} \frac{\rho\left(n_{\mathfrak{b}}-p\right)}{Z\left(n_{\mathfrak{b}}-p\right)}
\end{aligned}
$$

Whence treating the small ratio $\rho\left(n_{\mathfrak{b}}\right) / Z\left(n_{\mathfrak{b}}\right)$ as a smooth function of the continuous variable $n$ we get for $n_{\mathfrak{b}} \gg 1$

$$
\tilde{\Lambda}_{i}(\mathbf{r}) \simeq-g^{n_{\mathfrak{b}}-n_{i}} \frac{d}{d n}\left[\frac{\rho(n)}{Z(n)}\right]_{n=n_{\mathfrak{b}}} \sum_{p=1}^{\infty} p g^{-p}
$$

at leading order in this small parameter. Therefore we may state that $\tilde{\Lambda}_{i}(\mathbf{r})=o\left(\frac{\rho\left(n_{i}\right)}{Z\left(n_{i}\right)}\right)$ because in the given case $n_{\mathfrak{b}}<n_{i}$ and so either $n_{\mathfrak{b}} \sim n_{i}$ or $n_{i}-n_{\mathfrak{b}} \gg 1$ and for such values of $n_{\mathfrak{b}}$ the magnitude of $\tilde{\Lambda}_{i}(\mathbf{r})$ is of exponential smallness: $\tilde{\Lambda}_{i}(\mathbf{r}) \sim g^{n_{\mathfrak{b}}-n_{i}}$. The given nonzero value of $\tilde{\Lambda}_{i}(\mathbf{r})$ is actually due to variations of the function $\rho(n) / Z(n)$ as the argument $n$ changes on scales of order unity, so in this case we have to set $\tilde{\Lambda}_{i}(\mathbf{r})=0$.

Summarizing the aforesaid we see that if the point $\mathbf{r}$ is located inside the fundamental domain $\mathfrak{M}_{i}$ corresponding to the branch $i$, i.e. $\Theta_{i}(\mathbf{r})=1$ then the branch $i$ belongs to the path $\mathbb{P}(\mathbf{r})$ and the value $\tilde{\Lambda}_{i}(\mathbf{r})=1$ at leading order in $\rho(n) / Z(n)$. Otherwise, when $\mathbf{r} \notin \mathfrak{M}_{i}$ and so $i \notin \mathbb{P}(\mathbf{r})$ the value $\Theta_{i}(\mathbf{r})=0$ and we have to set $\tilde{\Lambda}_{i}(\mathbf{r})=0$. This substantiates identity $(\mathrm{B} .4)$.

\section{REFERENCES}


[1] G. I. Mchedlishvili, Microcirculation of Blood . General Principles of Control and Disturbances, Nauka, Leningrad, 1989 (in Russian).

[2] Heat Transfer in Medicine and Biology, Anal. and Appl (Vol. 1, 2), A. Shitzer and R. C. Eberhart, eds., Plenum, New York, 1985.

[3] Reading in Microeconomics, W. Breit, H. M. Hodrman, and E. Saueracker, eds., Times Mirror \& Mosby College Publishing, 1986.

[4] H. T. Odum, Environment, Power and Society, John Wiley \& Sons, New York, 1971.

[5] K. E. Boulding, The unimportance of energy, in Energetics and Systems, W. J. Mitsch, R. K. Ragade, R. W. Bosserman, and J.A.Dillon, eds., Ann Arbor Science, Ann Arbor, Michigan, 1982, pp. 101-108.

[6] S. M. Guriev, I. G. Pospelov, and M. B. Shahova, Model imitating self-organisation of trade networks, Preprint, Computing Center of Russian Academy of Sciences (1996) (in Russian).

[7] R. E. Ulanowicz, Growth and Development: Ecosystems Phenomenology, Springer-Verlag, New York, 1986.

[8] H. Sakaguchi, Hierarchical structures in Lotka-Volterra type equations, Physics Letters A, 196 (1994), pp. 38-42.

[9] S. E. Jorgensen, Integration of Ecosystem Theories: A Pattern, Kluwer Academic Publishers, 1992.

[10] P. A. Samuelson, Foundations of Economic Analysis, Harvard University Press, Cambridge, 1947.

[11] G. Nicolis And I. Prigogine, Self-Organization in Nonequilibrium Systems, John Wiley \& Sons, New York, 1977. 